\title{
Small time asymptotics of diffusion processes
}

\author{
A.F.M. ter Elst ${ }^{1}$, Derek W. Robinson ${ }^{2}$ and Adam Sikora ${ }^{3}$
}

\begin{abstract}
We establish the short-time asymptotic behaviour of the Markovian semigroups associated with strongly local Dirichlet forms under very general hypotheses. Our results apply to a wide class of strongly elliptic, subelliptic and degenerate elliptic operators. In the degenerate case the asymptotics incorporate possible non-ergodicity.
\end{abstract}

November 2005

AMS Subject Classification: 35B40, 58J65, 35J70, 35Hxx, 60J60.

Home institutions:

1. Department of Mathematics and Computing Science

Eindhoven University of Technology

P.O. Box 513

5600 MB Eindhoven

The Netherlands

3. Department of Mathematical Sciences

New Mexico State University

P.O. Box 30001

Las Cruces

NM 88003-8001, USA
2. Centre for Mathematics and its Applications Mathematical Sciences Institute Australian National University Canberra, ACT 0200

Australia 


\section{Introduction}

One of the iconic results in the theory of second-order elliptic operators is Varadhan's Var67b] Var67a identification of the small time asymptotic limit

$$
\lim _{t \downarrow 0} t \log K_{t}(x ; y)=-4^{-1} d(x ; y)^{2}
$$

of the heat kernel $K$ of a strongly elliptic operator on $\mathbf{R}^{d}$ in terms of the intrinsic Riemannian distance $d(\cdot ; \cdot)$. The small time behaviour was subsequently analyzed at length by Molchanov [Mol75] who extended (11) to a much wider class of operators and manifolds. These results were then analyzed, largely by probabilistic methods, by various authors (see, for example, the Paris lectures [Aze81]). Most of the early results were restricted to non-degenerate operators with smooth coefficients acting on smooth manifolds. Optimal results for the heat flow on Lipschitz Riemannian manifolds were obtained much later by Norris [Nor97]. The asymptotic relation (11) has, however, been established for certain classes of degenerate subelliptic operators by several authors, in particular for sublaplacians constructed from vector fields satisfying Hörmander's condition for hypoellipticity BKRR71 [Léa87a Léa87b KS88. It is nevertheless evident from explicit examples that (11) fails for large classes of degenerate elliptic operators. Difficulties arise, for example, from non-ergodic behaviour.

The problems introduced by degeneracies are illustrated by the operator $H=-d c_{\delta} d$, where $d=d / d x$ and

$$
c_{\delta}(x)=\left(\frac{x^{2}}{1+x^{2}}\right)^{\delta}
$$

acting on the real line. If $\delta \in[0,1 / 2\rangle$ then the associated diffusion is ergodic. If, however, $\delta \geq 1 / 2$ then the diffusion process separates into two processes on the left and right half lines, respectively (see ERSZ04, Proposition 6.5). The degeneracy of $c_{\delta}$ at the origin creates an impenetrable obstacle for the diffusion. Therefore the corresponding kernel $K$ satisfies $K_{t}(x ; y)=0$ for all $x<0, y>0$ and $t>0$ and the effective distance between the left and right half lines is infinite. But this behaviour is not reflected by the Riemannian distance $d(x ; y)=\left|\int_{x}^{y} c_{\delta}^{-1 / 2}\right|$ which is finite for all $\delta \in[0,1\rangle$. Hence (11) must fail for this diffusion process if $\delta \in[1 / 2,1\rangle$. More complicated phenomena can occur for degenerate operators in higher dimensions. To be specific let $I=\{(\alpha, 0): \alpha \in[-1,1]\}$ be a bounded one-dimensional interval in $\mathbf{R}^{2}$ and consider the form $h(\varphi)=\sum_{i=1}^{2}\left(\partial_{i} \varphi, c_{\delta} \partial_{i} \varphi\right)$ with $D(h)=W^{1,2}\left(\mathbf{R}^{2}\right)$ where $c_{\delta}(x)=\left(|x|_{I}^{2} /\left(1+|x|_{I}^{2}\right)\right)^{\delta}$ and $|x|_{I}$ denotes the Euclidean distance from $x \in \mathbf{R}^{2}$ to the interval $I$. It follows that if $\delta \geq 1 / 2$ then the interval $I$ presents an impenetrable obstacle for the corresponding diffusion and the effective configuration space for the process is $\mathbf{R}^{2} \backslash I$. In particular the appropriate distance for the description of the diffusion is the intrinsic Riemannian distance on $\mathbf{R}^{2} \backslash I$ rather than that on $\mathbf{R}^{2}$. Although the Riemannian distance on $\mathbf{R}^{2}$ is well-defined if $\delta \in[0,1\rangle$ it is not suited to the description of the diffusion if $\delta \in[1 / 2,1\rangle$. Thus the problem of a deeper understanding of the small time behaviour of the heat kernel associated with degenerate operators consists in part in identifying the appropriate measure of distance.

Hino and Ramírez HR03 (see also Hin02 Ram01) made considerable progress in understanding the small time asymptotics of general diffusion processes by examining the problem in the broader context of Dirichlet forms on a $\sigma$-finite measure space $(X, \mathcal{B}, \mu)$ [FOT94 [BH91] Mos94. First they consider an integrated version of (11). Set $K_{t}(A ; B)=$ 
$\int_{A} d \mu(x) \int_{B} d \mu(y) K_{t}(x ; y)$ for measurable subsets $A, B \in \mathcal{B}$. Then the pointwise asymptotic estimate (10) leads, under quite general conditions, to a set-theoretic version

$$
\lim _{t \downarrow 0} t \log K_{t}(A ; B)=-4^{-1} d(A ; B)^{2}
$$

for open sets where the distance between the sets $A$ and $B$ is defined in the usual manner with infima. Secondly, if the measure $\mu$ is finite then Hino-Ramírez establish (3) for the kernel of the semigroup corresponding to a strongly local, conservative, Dirichlet form on $L_{2}(X ; \mu)$ and for bounded measurable sets $A$ and $B$ but with a set-theoretic distance $d(A ; B)$ defined directly in terms of the Dirichlet form. This distance takes values in $[0, \infty]$ and is not necessarily the distance arising from any underlying Riemannian structure. Nevertheless the estimate (3) establishes that it is the correct measure of small time behaviour. A key feature of this formalism is that it allows for the possibility that $K_{t}(A ; B)=0$ for all small $t$ and then $d(A ; B)=\infty$. The principal disadvantages of the Hino-Ramírez result is that it requires $(X, \mathcal{B}, \mu)$ to be a probability space and the form to be conservative. One of our aims is to remove these restrictions and to derive the estimate (3) for diffusion processes related to a large class of regular, strongly local, Markovian forms on a general measure space $(X, \mathcal{B}, \mu)$. Our formalism is suited to applications to degenerate elliptic operators. A second aim is to establish conditions which allow one to pass from the estimate (3) to the pointwise estimate (11). We prove a general result which covers a variety of canonical situations. For example, we are able to derive pointwise estimates for subelliptic operators on Lie groups, to give an independent proof of Norris' result [Nor97] for the Laplace-Beltrami operator on a Lipschitz Riemannian manifold and to discuss Schrödinger-like semigroups with locally bounded potentials.

Simultaneous with this work Ariyoshi and Hino AH05] extended the Hino-Ramírez result to strongly local Dirichlet forms on general $\sigma$-finite measure spaces. Their tactic is broadly similar to ours although both were developed independently. Both proofs rely on local approximations and limits of the local diffusions and the corresponding distances. The limits are controlled by use of the evolution equation associated with the form. Ariyoshi and Hino refine the estimates on the equations of motion given in [HR03, and Ram01] whilst our arguments rely on the observation that the corresponding wave equation has a finite speed of propagation ERSZ04. The latter property gives rather precise information about the small scale evolution.

In Section 2 we establish the general formalism in which we work and give a complete definition of the distance $d(A ; B)$. Then we are able to give a precise statement of our conclusions. Proofs are given in Section 3. In Section 4 we discuss various applications to elliptic and subelliptic differential operators.

\section{General formalism}

We begin by summarizing some standard definitions and results on Markovian forms and Dirichlet forms. As background we refer to the books by Fukushima, Oshima and Takeda [FOT94, Bouleau and Hirsch [BH91] and Ma and Röckner [MR92] and the papers by LeJan [LeJ78] and Mosco [Mos94]. Most of the early results we need are summarized in Mosco Mos94 together with more recent results, notably on relaxed forms and convergence properties. 
Let $X$ be a connected locally compact separable metric space equipped with a positive Radon measure $\mu$, such that $\operatorname{supp} \mu=X$. We consider forms and operators on the real Hilbert space $L_{2}(X)$ and all functions in the sequel are assumed to be real-valued. Moreover, all forms are assumed to be symmetric and densely defined but not necessarily closed or even closable. We adopt the definition of Markovian form given in [FOT94, page 4, or Mosco [Mos94, page 374. Then a Dirichlet form is a closed Markovian form.

A Dirichlet form $\mathcal{E}$ on $L_{2}(X)$ is called regular if there is a subset of $D(\mathcal{E}) \cap C_{c}(X)$ which is a core of $\mathcal{E}$, i.e., which is dense in $D(\mathcal{E})$ with respect to the natural norm $\varphi \mapsto$ $\left(\mathcal{E}(\varphi)+\|\varphi\|_{2}^{2}\right)^{1 / 2}$, and which is also dense in $C_{0}(X)$ with respect to the supremum norm. There are several non-equivalent kinds of locality for forms or Dirichlet forms [BH91] FOT94 Mos94. In general we will call any positive quadratic form $h$ strongly local if $h(\psi, \varphi)=0$ for all $\varphi, \psi \in D(h)$ with $\operatorname{supp} \varphi$ and $\operatorname{supp} \psi$ compact and $\psi$ constant on a neighbourhood of $\operatorname{supp} \varphi$. Following Mosco [Mos94 we refer to a strongly local regular Dirichlet form as a diffusion.

There is a possibly stronger version of the locality condition given in BH91, Section I.5. A Dirichlet form $\mathcal{E}$ is called [BH]-local if $\mathcal{E}(\psi, \varphi)=0$ for all $\varphi, \psi \in D(\mathcal{E})$ and $a \in \mathbf{R}$ such that $(\varphi+a \mathbb{1}) \psi=0$. It follows, however, by [BH91] Remark I.5.1.5 and Proposition I.5.1.3 $\left(L_{0}\right) \Rightarrow\left(L_{2}\right)$, that strong locality and [BH]-locality are equivalent for regular Dirichlet forms. Therefore diffusions are $[\mathrm{BH}]$-local.

The Dirichlet form $\mathcal{E}$ is called conservative if $\mathbb{1} \in D(\mathcal{E})$ and $\mathcal{E}(\mathbb{1})=0$. The condition $\mathbb{1} \in D(\mathcal{E})$ of course requires that $\mathbb{1} \in L_{2}(X)$, i.e., $\mu(X)<\infty$, so it is of limited applicability.

Let $\mathcal{E}$ be a general Dirichlet form on $L_{2}(X)$. First, for all $\psi \in D(\mathcal{E}) \cap L_{\infty}(X)$ define $\mathcal{I}_{\psi}^{(\mathcal{E})}: D(\mathcal{E}) \cap L_{\infty}(X) \rightarrow \mathbf{R}$ by

$$
\mathcal{I}_{\psi}^{(\mathcal{E})}(\varphi)=\mathcal{E}(\psi \varphi, \psi)-2^{-1} \mathcal{E}\left(\psi^{2}, \varphi\right)
$$

If no confusion is possible we drop the suffix and write $\mathcal{I}_{\psi}(\varphi)=\mathcal{I}_{\psi}^{(\mathcal{E})}(\varphi)$. If $\varphi \geq 0$ it follows that $\psi \mapsto \mathcal{I}_{\psi}(\varphi)$ is a Markovian form with domain $D(\mathcal{E}) \cap L_{\infty}(X)$ which satisfies the key properties

$$
0 \leq \mathcal{I}_{F \circ \psi}(\varphi) \leq \mathcal{I}_{\psi}(\varphi) \leq\|\varphi\|_{\infty} \mathcal{E}(\psi)
$$

for all $\varphi, \psi \in D(\mathcal{E}) \cap L_{\infty}(X)$ with $\varphi \geq 0$ and all normal contractions $F$ (see [BH91], Proposition I.4.1.1). Hence

$$
\left|\mathcal{I}_{\psi_{1}}(\varphi)-\mathcal{I}_{\psi_{2}}(\varphi)\right| \leq \mathcal{I}_{\psi_{1}-\psi_{2}}(\varphi)^{1 / 2} \mathcal{I}_{\psi_{1}+\psi_{2}}(\varphi)^{1 / 2} \leq \mathcal{E}\left(\psi_{1}-\psi_{2}\right)^{1 / 2} \mathcal{E}\left(\psi_{1}+\psi_{2}\right)^{1 / 2}\|\varphi\|_{\infty}
$$

for all $\varphi, \psi_{1}, \psi_{2} \in D(\mathcal{E}) \cap L_{\infty}(X)$ with $\varphi \geq 0$. This form is referred to as the truncated form by Roth [Rot76], Theorem 5 .

A regular Dirichlet form has a canonical representation originating with Beurling and Deny [BD58] and in its final form by LeJan [LeJ78, (see [Mos94, Section 3e or [FOT94, Section 3.2). If $\mathcal{E}$ is strongly local, i.e., if it is a diffusion, this representation takes the simple form

$$
\mathcal{E}(\psi)=\int_{X} d \mu_{\psi}(x)
$$

for all $\psi \in D(\mathcal{E})$ where the $\mu_{\psi}\left(=\mu_{\psi}^{(\mathcal{E})}\right)$ are positive Radon measures. The measures are uniquely determined by the identity

$$
\mathcal{I}_{\psi}(\varphi)=\int_{X} d \mu_{\psi} \varphi
$$


for all $\varphi, \psi \in D(\mathcal{E}) \cap L_{\infty}(X)$ and the continuity property

$$
\left\|\mu_{\psi_{1}}-\mu_{\psi_{2}}\right\| \leq \mathcal{E}\left(\psi_{1}-\psi_{2}\right)^{1 / 2} \mathcal{E}\left(\psi_{1}+\psi_{2}\right)^{1 / 2}
$$

for all $\psi_{1}, \psi_{2} \in D(\mathcal{E})$ as a consequence of (15) (see [LeJ78], Propositions 1.4.1 and 1.5.1 or [FOT94, Section 3.2 and Lemma 3.2.3). The measure $\mu_{\psi}$ is usually referred to as the energy measure.

If $\mathcal{E}$ and $\mathcal{F}$ are two Dirichlet forms which are [BH]-local and satisfy $\mathcal{E} \leq \mathcal{F}$ then

$$
\mathcal{I}_{\psi}^{(\mathcal{E})}(\varphi) \leq \mathcal{I}_{\psi}^{(\mathcal{F})}(\varphi)
$$

for all $\varphi, \psi \in D(\mathcal{F}) \cap L_{\infty}(X)$ with $\varphi \geq 0$ (see, for example, ERSZ05] Proposition 3.2).

Let $\mathcal{E}$ be a diffusion. Define $D(\mathcal{E})_{\text {loc }}$ as the vector space of (equivalent classes of) all measurable functions $\psi: X \rightarrow \mathbf{C}$ such that for every compact subset $K$ of $X$ there is a $\hat{\psi} \in D(\mathcal{E})$ with $\left.\psi\right|_{K}=\left.\hat{\psi}\right|_{K}$. Since $\mathcal{E}$ is regular and $[\mathrm{BH}]$-local one can define $\widehat{\mathcal{I}}_{\psi}^{(\mathcal{E})}=$ $\widehat{\mathcal{I}}_{\psi}: D(\mathcal{E}) \cap L_{\infty, c}(X) \rightarrow \mathbf{R}$ by

$$
\widehat{\mathcal{I}}_{\psi}(\varphi)=\mathcal{I}_{\hat{\psi}}(\varphi)
$$

for all $\psi \in D(\mathcal{E})_{\text {loc }} \cap L_{\infty}(X)$ and $\varphi \in D(\mathcal{E}) \cap L_{\infty, c}(X)$ where $\hat{\psi} \in D(\mathcal{E}) \cap L_{\infty}(X)$ is such that $\left.\psi\right|_{\operatorname{supp} \varphi}=\left.\hat{\psi}\right|_{\operatorname{supp} \varphi}$. Next, for all $\psi \in D(\mathcal{E})_{\operatorname{loc}} \cap L_{\infty}(X)$, define

$$
\left\|\mid \widehat{\mathcal{I}}_{\psi}\right\| \|=\sup \left\{\left|\widehat{\mathcal{I}}_{\psi}(\varphi)\right|: \varphi \in D(\mathcal{E}) \cap L_{\infty, c}(X),\|\varphi\|_{1} \leq 1\right\} \in[0, \infty] .
$$

Now, for all $\psi \in L_{\infty}(X)$ and measurable sets $A, B \subset X$, introduce

$$
\begin{aligned}
d_{\psi}(A ; B) & =\sup \{M \in \mathbf{R}: \psi(x)-\psi(y) \geq M \text { for a.e. } x \in A \text { and a.e. } y \in B\} \\
& =\underset{x \in A}{\operatorname{essinf}} \psi(x)-\underset{y \in B}{\operatorname{essipup}} \psi(y) \in\langle-\infty, \infty] .
\end{aligned}
$$

Recall that

$$
\begin{aligned}
\underset{y \in B}{\operatorname{essup}} \psi(y) & =\inf \{m \in \mathbf{R}:|\{y \in B: \psi(y)>m\}|=0\} \\
& =\min \{m \in[-\infty, \infty\rangle:|\{y \in B: \psi(y)>m\}|=0\} \in[-\infty, \infty\rangle
\end{aligned}
$$

and ess $\inf _{x \in A} \psi(x)=-\operatorname{ess}_{\sup _{x \in A}}-\psi(x)$. Finally define

$$
d(A ; B)=d^{(\mathcal{E})}(A ; B)=\sup \left\{d_{\psi}(A ; B): \psi \in D_{0}(\mathcal{E})\right\},
$$

where

$$
D_{0}(\mathcal{E})=\left\{\psi \in D(\mathcal{E})_{\text {loc }} \cap L_{\infty}(X):\left\|\widehat{\mathcal{I}}_{\psi}\right\| \| \leq 1\right\}
$$

as in [ERSZ05]. A similar definition was given by Hino and Ramírez HR03] (see also [Stu98]), but since they considered probability spaces the introduction of $D(\mathcal{E})_{\text {loc }}$ was unnecessary. Regularity of the form was also unnecessary. If, however, we were to replace $D(\mathcal{E})_{\text {loc }}$ by $D(\mathcal{E})$ in the definition of $d(A ; B)$ then, since $\psi \in L_{2}(X)$, one would obtain $d_{\psi}(A ; B) \leq 0$ for all measurable sets $A, B \subset X$ with $|A|=|B|=\infty$ and this would give $d(A ; B)=0$. On the other hand the definition with $D(\mathcal{E})_{\text {loc }}$ is not useful unless $D(\mathcal{E})$ contains sufficient bounded functions with compact support. Hence regularity of some sort is essential.

The distance determines the following $L_{2}$-off diagonal bounds, or Davies-Gaffney bounds on the semigroup associated to a diffusion by Theorem 1.2 in [ERSZ05]. 
Theorem 2.1 Let $\mathcal{E}$ be a diffusion over $X$. If $S^{(\mathcal{E})}$ denotes the semigroup generated by the self-adjoint operator $H$ on $L_{2}(X)$ associated with $\mathcal{E}$ and if $A$ and $B$ are measurable subsets of $X$ then

$$
\left|\left(\psi, S_{t}^{(\mathcal{E})} \varphi\right)\right| \leq e^{-d^{(\mathcal{E})}(A ; B)^{2}(4 t)^{-1}}\|\psi\|_{2}\|\varphi\|_{2}
$$

for all $\psi \in L_{2}(A), \varphi \in L_{2}(B)$ and $t>0$ with the convention $e^{-\infty}=0$.

Let $h$ be a positive quadratic form on $L_{2}(X)$. Then $h$ is not necessarily closable but there exists a largest closed quadratic form, denoted by $h_{0}$, which is majorized by $h$, i.e., $D(h) \subseteq D\left(h_{0}\right)$ and $h_{0}(\varphi) \leq h(\varphi)$ for all $\varphi \in D(h)$. The form $h_{0}$ is referred to in the literature on discontinuous media as the relaxation of $h$ (see [Mos94, Section 1.e). It can be understood in various ways. Simon (see Sim78, Theorems 2.1 and 2.2) established that $h$ can be decomposed uniquely as a sum $h=h_{r}+h_{s}$ of two positive forms with $D\left(h_{r}\right)=D(h)=D\left(h_{s}\right)$ and $h_{r}$ the largest closable form majorized by $h$. Simon refers to $h_{r}$ as the regular part of $h$. Then $h_{0}=\overline{h_{r}}$, the closure of $h_{r}$. Simon also proved that $D\left(h_{0}\right)$ consists of those $\varphi \in L_{2}(X)$ for which there is a sequence $\varphi_{n} \in D(h)$ such that $\lim _{n \rightarrow \infty} \varphi_{n}=\varphi$ in $L_{2}(X)$ and $\liminf _{n \rightarrow \infty} h\left(\varphi_{n}\right)<\infty$. Moreover, $h_{0}(\varphi)$ equals the minimum of all $\liminf \operatorname{in}_{n \rightarrow \infty} h\left(\varphi_{n}\right)$, where the minimum is taken over all $\varphi_{1}, \varphi_{2}, \ldots \in D(h)$ such that $\lim _{n \rightarrow \infty} \varphi_{n}=\varphi$ in $L_{2}(X)$. (See [Sim77, Theorems 2 and 3.) Note that if $D$ is a subspace of $L_{2}(X)$ which is dense in $D(h)$ then $\left(\left.h\right|_{D}\right)_{0}=h_{0}$. Moreover, if $h$ and $k$ are two positive quadratic forms with $h \leq k$ then $h_{0} \leq k_{0}$.

The relaxation can also be understood by approximation. If $h, h_{1}, h_{2}, \ldots$ are closed positive quadratic forms on $L_{2}(X)$ and $H, H_{1}, H_{2}, \ldots$ the corresponding positive self-adjoint operators then we write $h=\mathrm{r}^{-\lim _{n \rightarrow \infty}} h_{n}$ if $H_{1}, H_{2}, \ldots$ converges to $H$ in the strong resolvent sense, i.e., if $(I+H)^{-1}=\lim _{n \rightarrow \infty}\left(I+H_{n}\right)^{-1}$ strongly. It follows from Mos94, Theorem 2.4.1, that $h=r \cdot \lim _{n \rightarrow \infty} h_{n}$ if and only if $h(\varphi) \leq \liminf _{n \rightarrow \infty} h_{n}\left(\varphi_{n}\right)$ for all $\varphi, \varphi_{1}, \varphi_{2}, \ldots \in L_{2}(X)$ with $\lim _{n \rightarrow \infty} \varphi_{n}=\varphi$ weakly in $L_{2}(X)$ and, in addition, for all $\varphi \in L_{2}(X)$ there exists a sequence $\varphi_{1}, \varphi_{2}, \ldots \in L_{2}(X)$ such that $\lim _{n \rightarrow \infty} \varphi_{n}=\varphi$ strongly in $L_{2}(X)$ and $h(\varphi)=\liminf _{n \rightarrow \infty} h_{n}\left(\varphi_{n}\right)$.

If $h_{1}, h_{2}, \ldots$ are closed positive quadratic forms on $L_{2}(X)$ such that $h_{1} \geq h_{2} \geq \ldots$ then it follows from a result of Kato Kat80, Theorem VIII.3.11, that the corresponding sequence $H_{1}, H_{2}, \ldots$ of operators converges in the strong resolvent sense to a positive selfadjoint operator $H$. If $h$ is the form corresponding to $H$, then $h=r \cdot \lim _{n \rightarrow \infty} h_{n}$ and $h$ is the largest closed form which is majorized by $h_{n}$ for all $n \in \mathbf{N}$ (Simon [Sim77], Theorem 3.2).

Now let $h$ be a positive quadratic form on $L_{2}(X)$ and $l$ a closed positive quadratic form such that $h \leq \lambda l$, for some $\lambda>0$, and with $D(l)$ dense in $D(h)$. Then the forms $h_{\varepsilon}=h+\varepsilon l$, with $\varepsilon>0$, are all closed positive forms, with domain $D(l)$, since $\varepsilon l \leq h_{\varepsilon} \leq(\lambda+\varepsilon) l$. But $\varepsilon \mapsto h_{\varepsilon}$ is monotonically decreasing if $\varepsilon \downarrow 0$ and it follows from the results of Kato and Mosco cited in the foregoing paragraphs that $r . \lim _{\varepsilon \downarrow 0} h_{\varepsilon}=h_{0}$ where $h_{0}$ is the relaxation of $h$. This characterization justifies the notation $h_{0}$. Note that $h_{0}$ is independent of the particular $l$ used in this construction which is akin to the viscosity method of partial differential equations. So $h_{0}$ could well be called the viscosity closure of $h$. If $h$ is the form of a pure second-order elliptic operator in divergence form on $\mathbf{R}^{d}$ and $l$ is the form of the Laplacian then the condition $h \leq \lambda l$ corresponds to uniform boundedness of the coefficients.

Throughout the remainder of this paper we fix a strongly local regular Dirichlet form $l$ on $L_{2}(X)$, i.e., a diffusion, satisfying the following property. 
Condition $\mathbf{L}$ The function $d^{(e)}: X \times X \rightarrow[0, \infty]$ defined by

$$
d^{(e)}(x ; y)=\sup \left\{|\psi(x)-\psi(y)|: \psi \in D(l)_{\text {loc }} \cap C_{\mathrm{b}}(X) \text { and }\left\|\widehat{\mathcal{I}}_{\psi}^{(l)}\right\| \mid \leq 1\right\}
$$

is a metric, the topology induced by this metric equals the original topology on $X$ and the balls $B^{(e)}(x ; r)$ defined by the metric $d^{(e)}$ are relatively compact for all $x \in X$ and $r>0$.

The first result of this paper is given by the following.

Theorem 2.2 Let $l$ be a diffusion on $L_{2}(X)$

satisfying Condition L. Further let $h$ be a positive form over $X$ such that $h \leq \lambda l_{D(l) \cap L_{\infty}(X)}$ for some $\lambda>0$ and $\left.h\right|_{D(l) \cap L_{\infty}(X)}$ is strongly local and Markovian.

Then the relaxation $h_{0}$ of $h$ is a diffusion. Moreover, if $A, B \subset X$ are relatively compact and measurable then

$$
\lim _{t \downarrow 0} t \log \left(\mathbb{1}_{A}, S_{t}^{\left(h_{0}\right)} \mathbb{1}_{B}\right)=-4^{-1} d^{\left(h_{0}\right)}(A ; B)^{2}
$$

where $S^{\left(h_{0}\right)}$ denotes the semigroup associated with $h_{0}$.

Note that if $S^{\left(h_{0}\right)}$ has an integrable kernel $K^{\left(h_{0}\right)}$ then

$$
\left(\mathbb{1}_{A}, S_{t}^{\left(h_{0}\right)} \mathbb{1}_{B}\right)=\int_{A} d \mu(x) \int_{B} d \mu(y) K_{t}^{\left(h_{0}\right)}(x ; y)=K_{t}^{\left(h_{0}\right)}(A ; B)
$$

so (17) corresponds to the integrated version (3) of Varadhan's result (11).

Next note that it follows by choosing $h$ equal to $l$ that one has the following conclusion.

Corollary 2.3 If $l$ is a diffusion on $L_{2}(X)$ satisfying Condition $\mathrm{L}$, then

$$
\lim _{t \downarrow 0} t \log \left(\mathbb{1}_{A}, S_{t}^{(l)} \mathbb{1}_{B}\right)=-4^{-1} d^{(l)}(A ; B)^{2}
$$

for all relatively compact measurable $A, B \subset X$.

One application of the corollary is to strongly elliptic operators in divergence form on $\mathbf{R}^{d}$ with bounded coefficients. Then $d^{(e)}(\cdot ; \cdot)$ is the corresponding Riemannian distance and $d^{(l)}(A ; B)=d^{(e)}(A ; B)$ if $A$ and $B$ are open and non-empty, where we set

$$
d^{(e)}(A ; B)=\inf _{x \in A} \inf _{y \in B} d^{(e)}(x ; y)
$$

for general non-empty $A, B \subseteq X$.

There is also a weaker version of Theorem 2.2 for sets which are possibly not relatively compact. In the formulation of this result we let $P_{A}$ denote the orthogonal projection from $L_{2}(X)$ onto $L_{2}(A)$.

Corollary 2.4 Assume the conditions of Theorem 2.2. Then

$$
\lim _{t \downarrow 0} t \log \left\|P_{A} S_{t}^{\left(h_{0}\right)} P_{B}\right\|_{2 \rightarrow 2}=-4^{-1} d^{\left(h_{0}\right)}(A ; B)^{2}
$$

for all measurable $A, B \subset X$. 
It is possible to transform the set-theoretic bounds on the semigroup into pointwise bounds but this requires some additional assumptions which are satisfied for large classes of non-degenerate elliptic operators (see Section 4). In general it is possible that $\psi \in$ $D(l)_{\text {loc }} \cap L_{\infty}$ with $\left\|\left|\widehat{\mathcal{I}}_{\psi} \|\right|<\infty\right.$ but $\psi$ is not continuous. Therefore one can have $d^{(l)}(A ; B)>$ $d^{(e)}(A ; B)$ even for non-empty open sets $A$ and $B$. This behaviour is illustrated by the one-dimensional example given in the introduction with $\delta \in[1 / 2,1\rangle$ in (2))

This explains the origin of the first assumption in the next theorem. The second assumption is typically a consequence of a parabolic version of the Harnack inequality.

Theorem 2.5 Let $l$ be a diffusion on $L_{2}(X)$ satisfying Condition L. Assume

I. $D_{0}(l) \subseteq C(X)$, and,

II. the semigroup $S^{(l)}$ has a continuous kernel $K$ and there are $\nu, \omega, T \in\langle 0, \infty\rangle$ such that

$$
K_{s}(x ; y) \leq K_{t}(x ; z)\left(t s^{-1}\right)^{\nu} e^{\omega\left(1+d^{(e)}(y ; z)^{2}(t-s)^{-1}\right)}
$$

for all $0<s<t \leq T$ and $x, y, z \in X$.

Then

$$
\lim _{t \downarrow 0} t \log K_{t}(x ; y)=-4^{-1} d^{(e)}(x ; y)^{2}
$$

for all $x, y \in X$.

Applications of Theorem 2.5] are discussed in Section 4.

\section{Proof of the theorems}

First we derive several useful results for general diffusions.

Lemma 3.1 Let $\mathcal{E}$ be a diffusion on $L_{2}(X)$. If $A, B$ are measurable with $|A|,|B|<\infty$ and

$$
\lim _{t \downarrow 0} t \log \left(\mathbb{1}_{A}, S_{t}^{(\mathcal{E})} \mathbb{1}_{B}\right)=-4^{-1} d^{(\mathcal{E})}(A ; B)^{2}
$$

then $d^{(\mathcal{E})}(A ; B)$ is the supremum of all $r \geq 0$ for which there are $M, t_{0}>0$ such that

$$
\left(\mathbb{1}_{A}, S_{t}^{(\mathcal{E})} \mathbb{1}_{B}\right) \leq M e^{-(4 t)^{-1} r^{2}}
$$

for all $t \in\left\langle 0, t_{0}\right]$.

Proof Let $s$ denote the supremum. Then the Davies-Gaffney bounds of Theorem 2.1 give $d(A ; B) \leq s$. If $d(A ; B)<s$ then there are $r \in\langle d(A ; B), s\rangle$ and $M, t_{0}>0$ such that $\left(\mathbb{1}_{A}, S_{t} \mathbb{1}_{B}\right) \leq M e^{-(4 t)^{-1} r^{2}}$ for all $t \in\left\langle 0, t_{0}\right]$. Then

$$
\lim _{t \downarrow 0} t \log \left(\mathbb{1}_{A}, S_{t} \mathbb{1}_{B}\right) \leq-4^{-1} r^{2}<-4^{-1} d(A ; B)^{2}
$$

which gives a contradiction.

Lemma 3.2 Let $\mathcal{E}$ be a diffusion on $L_{2}(X)$. Let $\psi_{1}, \psi_{2} \in D(\mathcal{E})_{\text {loc }} \cap L_{\infty}$. 
I. If $\varphi \in D(\mathcal{E}) \cap L_{\infty, c}$ with $\varphi \geq 0$ then $\widehat{\mathcal{I}}_{\psi_{1} \wedge \psi_{2}}^{(\mathcal{E})}(\varphi) \leq \widehat{\mathcal{I}}_{\psi_{1}}^{(\mathcal{E})}(\varphi)+\widehat{\mathcal{I}}_{\psi_{1}}^{(\mathcal{E})}(\varphi)$.

II. $\quad\left\|\widehat{\mathcal{I}}_{\psi_{1} \vee \psi_{2}}^{(\mathcal{E})}\right\|\left|\leq\left\|\widehat{\mathcal{I}}_{\psi_{1}}^{(\mathcal{E})}\left|\|\vee\| \widehat{\mathcal{I}}_{\psi_{2}}^{(\mathcal{E})} \|\right|\right.\right.$.

Proof Let $\mu_{\psi}$ be the energy measure associated with $\mathcal{E}$. It follows from $(2.10)$ in [BM95] that

$$
\mu_{\psi_{1} \wedge \psi_{2}}=\mathbb{1}_{\left\{x \in X: \psi_{1}(x)<\psi_{2}(x)\right\}} \mu_{\psi_{1}}+\mathbb{1}_{\left\{x \in X: \psi_{1}(x)>\psi_{2}(x)\right\}} \mu_{\psi_{2}}
$$

for all $\psi_{1}, \psi_{2} \in D(l) \cap L_{\infty}$. Hence

$$
\begin{aligned}
\mathcal{I}_{\psi_{1} \wedge \psi_{2}}(\varphi) & =\int d \mu_{\psi_{1} \wedge \psi_{2}} \varphi=\int d \mu_{\psi_{1}} \varphi \mathbb{1}_{\left\{x \in X: \psi_{1}(x)<\psi_{2}(x)\right\}}+\int d \mu_{\psi_{2}} \varphi \mathbb{1}_{\left\{x \in X: \psi_{1}(x)>\psi_{2}(x)\right\}} \\
& \leq \int d \mu_{\psi_{1}} \varphi+\int d \mu_{\psi_{2}} \varphi=\mathcal{I}_{\psi_{1}}(\varphi)+\mathcal{I}_{\psi_{1}}(\varphi)
\end{aligned}
$$

for all $\varphi \in D(\mathcal{E}) \cap L_{\infty}$ with $\varphi \geq 0$. Now Statement $\llbracket$ of the lemma follows.

The proof of Statement $\amalg$ is similar.

Lemma 3.3 Let $\mathcal{E}$ be a diffusion on $L_{2}(X)$. Let $\psi_{1}, \psi_{2}, \ldots: X \rightarrow[0, \infty\rangle$ be measurable and assume that $\psi_{n} \wedge N \in D_{0}(\mathcal{E})$ for all $n, N \in \mathbf{N}$. Define $\psi: X \rightarrow[0, \infty]$ by $\psi=\sup _{n \in \mathbf{N}} \psi_{n}$. Then $\psi \wedge N \in D_{0}(\mathcal{E})$ for all $N \in \mathbf{N}$.

Proof Since $\varphi_{1} \vee \varphi_{2} \in D_{0}(\mathcal{E})$ for all $\varphi_{1}, \varphi_{2} \in D_{0}(\mathcal{E})$ by Lemma 3.2III we may assume that $\psi_{1} \leq \psi_{2} \leq \ldots$. Let $N \in \mathbf{N}$. We may also assume that $\psi_{n} \leq N$ for all $n \in \mathbf{N}$ by (44).

Let $K \subset X$ compact. Since $\mathcal{E}$ is regular there exist $\chi, \tilde{\chi} \in D(\mathcal{E}) \cap C_{c}(X)$ such that $0 \leq \chi \leq \tilde{\chi} \leq 1,\left.\chi\right|_{K}=1$ and $\left.\tilde{\chi}\right|_{\operatorname{supp} \chi}=1$. It follows from Lemma 3.2I and the strong locality of $\mathcal{E}$ that

$$
\mathcal{E}\left(\psi_{n} \wedge N \chi\right)=\mathcal{I}_{\psi_{n} \wedge N \chi}(\tilde{\chi}) \leq \widehat{\mathcal{I}}_{\psi_{n}}(\tilde{\chi})+\mathcal{I}_{N \chi}(\tilde{\chi}) \leq\|\tilde{\chi}\|_{1}+N^{2} \mathcal{E}(\chi)
$$

uniformly for all $n \in \mathbf{N}$. Moreover, $\left\|\psi_{n} \wedge N \chi\right\|_{2} \leq N\|\chi\|_{2}$ uniformly for all $n \in \mathbf{N}$. Hence the sequence $\psi_{1} \wedge N \chi, \psi_{2} \wedge N \chi, \ldots$ is bounded in $D(\mathcal{E})$. Therefore there exists a subsequence such that $\lim _{k \rightarrow \infty} \psi_{n_{k}} \wedge N \chi$ exists weakly in $D(\mathcal{E})$ and $\lim _{k \rightarrow \infty} \psi_{n_{k}} \wedge N \chi$ exists almost everywhere. By definition of $\psi$ one has $\lim _{k \rightarrow \infty} \psi_{n_{k}} \wedge N \chi=\psi \wedge N \chi$ almost everywhere. Hence $\psi \wedge N \chi \in D(\mathcal{E})$. In particular $\psi \in D(\mathcal{E})_{\text {loc }} \cap L_{\infty}$. Moreover, it follows from Statement (a) on page 269 of $\operatorname{Sim} 77$ ] that

$$
\begin{aligned}
\widehat{\mathcal{I}}_{\psi}(\varphi)=\mathcal{I}_{\psi \wedge N \chi}(\varphi) & \leq \liminf _{k \rightarrow \infty} \mathcal{I}_{\psi_{n_{k}} \wedge N \chi}(\varphi) \\
& =\liminf _{k \rightarrow \infty} \widehat{\mathcal{I}}_{\psi_{n_{k}}}(\varphi) \leq \liminf _{k \rightarrow \infty}\left\|\widehat{\mathcal{I}}_{\psi_{n_{k}}}\right\|\|\varphi\|_{1} \leq\|\varphi\|_{1}
\end{aligned}
$$

for all $\varphi \in D(\mathcal{E}) \cap L_{\infty, c}$ with $\operatorname{supp} \varphi \subset K$ and $\varphi \geq 0$. So $\psi \in D_{0}(\mathcal{E})$.

Lemma 3.4 Let $\mathcal{E}$ be a diffusion on $L_{2}(X)$. Let $X_{1} \subset X_{2} \subset \ldots$ be measurable subsets of $X$ such that $X=\bigcup_{n=1}^{\infty} X_{n}$. If $A, B$ are measurable subsets of $X$ then

$$
d^{(\mathcal{E})}(A ; B)=\lim _{n \rightarrow \infty} d^{(\mathcal{E})}\left(A \cap X_{n} ; B\right)=\inf _{n \in \mathbf{N}} d^{(\mathcal{E})}\left(A \cap X_{n} ; B\right) .
$$


Proof Obviously $d\left(A \cap X_{1} ; B\right) \geq d\left(A \cap X_{2} ; B\right) \geq \ldots \geq d(A ; B)$. Hence $\lim _{n \rightarrow \infty} d(A \cap$ $\left.X_{n} ; B\right)=\inf _{n \rightarrow \infty} d\left(A \cap X_{n} ; B\right) \geq d(A ; B)$. Let $M \in[0, \infty\rangle$ and suppose that $M \leq$ $\inf _{n \in \mathbf{N}} d\left(A \cap X_{n} ; B\right)$.

Let $\varepsilon>0$. Then by Lemma 4.2.III in ERSZ05 for all $n \in \mathbf{N}$ there exists a $\psi_{n} \in D_{0}(\mathcal{E})$ such that $0 \leq \psi_{n} \leq M,\left.\psi_{n}\right|_{B}=0$ and $\left.\psi_{n}\right|_{A \cap X_{n}} \geq M-\varepsilon$. Set $\psi=\sup _{n \in \mathbf{N}} \psi_{n}$. Then $\psi \in D_{0}(\mathcal{E})$ by Lemma 3.3. Moreover, $\left.\psi\right|_{A} \geq M-\varepsilon$ and $\left.\psi\right|_{B}=0$. So

$$
M-\varepsilon \leq d_{\psi}(A ; B) \leq d(A ; B)
$$

Since this is valid for all $M \in[0, \infty\rangle$ with $M \leq \inf _{n \in \mathbf{N}} d\left(A \cap X_{n} ; B\right)$ and all $\varepsilon>0$ it follows that $d(A ; B) \geq \inf _{n \in \mathbf{N}} d\left(A \cap X_{n} ; B\right)$ and the proof of the lemma is complete.

Next we develop some structural results which depend on the domination property assumed in Theorem 2.2. Initially we do not need to assume that $l$ satisfies Condition L.

We fix from now on a diffusion $l$ over $X$ and set $\mathfrak{A}=D(l) \cap L_{\infty}(X)$. Define $\mathcal{C}_{l}$ as the cone of all positive quadratic forms $h$ over $X$ with $\mathfrak{A} \subseteq D(h)$ satisfying

1. $h \leq\left.\lambda l\right|_{\mathfrak{A}}$ for some $\lambda>0$,

2. $\mathfrak{A}$ is dense in $D(h)$, and,

3. $\left.h\right|_{\mathfrak{A}}$ is strongly local and Markovian.

Note that we do not assume that the forms $h$ are closed or even closable.

The first lemma shows that there is no confusion possible for $h(\varphi)$ if $\varphi \in D(l) \backslash \mathfrak{A}$ under a mild condition that is satisfied if $h \in \mathcal{C}_{l}$.

Lemma 3.5 Let $h$ be a positive quadratic form over $X$ with $D(h)=\mathfrak{A}$ and suppose there

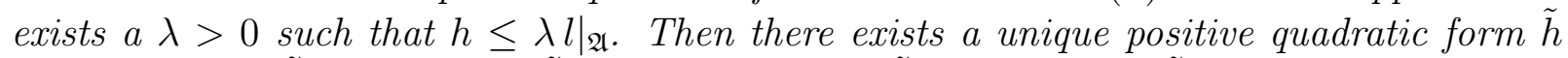
over $X$ with $D(\tilde{h})=D(l)$ and $\left.\tilde{h}\right|_{\mathfrak{A}}=h$. This form $\tilde{h}$ then satisfies $\tilde{h} \leq \lambda l$.

Proof The existence and the second part of the lemma are easy.

So it remains to prove the uniqueness. Let $\tilde{h}, \hat{h}$ be two positive quadratic form over $X$ with $D(\tilde{h})=D(l)=D(\hat{h})$ and $\left.\tilde{h}\right|_{\mathfrak{A}}=h=\left.\hat{h}\right|_{\mathfrak{A}}$. Let $\varepsilon>0$. Set

$$
\tilde{h}_{\varepsilon}=(1+\varepsilon)^{-1}(\tilde{h}+\varepsilon \lambda l) \quad \text { and } \quad \hat{h}_{\varepsilon}=(1+\varepsilon)^{-1}(\hat{h}+\varepsilon \lambda l)
$$

Then $D\left(\tilde{h}_{\varepsilon}\right)=D(l)=D\left(\hat{h}_{\varepsilon}\right)$ and $\tilde{h}_{\varepsilon}(\varphi)=\hat{h}_{\varepsilon}(\varphi)$ for all $\varphi \in \mathfrak{A}$. Moreover, $\tilde{h}_{\varepsilon}(\varphi) \leq \lambda l(\varphi)$ for all $\varphi \in \mathfrak{A}$. Let $\varphi \in D(l)$. Since $\mathfrak{A}$ is dense in $D(l)$ there are $\varphi_{1}, \varphi_{2}, \ldots \in \mathfrak{A}$ such that $\lim _{n \rightarrow \infty}\left(l\left(\varphi-\varphi_{n}\right)+\left\|\varphi-\varphi_{n}\right\|_{2}\right)=0$. Since $\tilde{h}_{\varepsilon}$ is closed and $\left.\tilde{h}\right|_{\mathfrak{A}} \leq\left. l\right|_{\mathfrak{A}}$ it follows from Kat80] Theorem VI.1.12, that $\tilde{h}_{\varepsilon}(\varphi)=\lim _{n \rightarrow \infty} \tilde{h}_{\varepsilon}\left(\varphi_{n}\right)$. But similarly $\hat{h}_{\varepsilon}(\varphi)=\lim _{n \rightarrow \infty} \hat{h}_{\varepsilon}\left(\varphi_{n}\right)$. Therefore $\tilde{h}_{\varepsilon}(\varphi)=\hat{h}_{\varepsilon}(\varphi)$. This is valid for all $\varepsilon>0$. Hence $\tilde{h}(\varphi)=\lim _{\varepsilon \downarrow 0} \tilde{h}_{\varepsilon}(\varphi)=\ldots=$ $\hat{h}_{\varepsilon}(\varphi)$.

Corollary 3.6 If $h \in \mathcal{C}_{l}$ then there is a unique $\tilde{h} \in \mathcal{C}_{l}$ such that $D(\tilde{h})=D(l)$ and $\left.\tilde{h}\right|_{\mathfrak{A}}=$ $\left.h\right|_{\mathfrak{A} \text {. }}$

It also follows from Lemma 3.5 that for a positive quadratic form $h$ over $X$ with $D(h) \subseteq D(l)$ one can give a characterization of the condition $h \in \mathcal{C}_{l}$. 
Corollary 3.7 If $h$ is a quadratic form over $X$ with $D(l) \subseteq D(h)$ and if $\lambda>0$ then the following are equivalent.

I. $\quad h \leq\left.\lambda l\right|_{\mathfrak{A}}$ and $\mathfrak{A}$ is dense in $D(h)$.

II. $\quad h \leq \lambda l$ and $D(l)$ is dense in $D(h)$.

Next we begin the analysis of the relaxations of the forms in $\mathcal{C}_{l}$.

Lemma 3.8 Let $h \in \mathcal{C}_{l}$. Then the relaxation $h_{0}$ of $h$ is a regular Dirichlet form with $D(l) \subseteq D\left(h_{0}\right)$. Moreover, every core for the form $l$ is a core for $h_{0}$.

Proof By assumption the algebra $\mathfrak{A}$ is dense in $D(h)$. Therefore $\left(\left.h\right|_{\mathfrak{A}}\right)_{0}=h_{0}$. Since $\left.h\right|_{\mathfrak{A}}$ is Markovian it follows from [Mos94, Corollary 2.8.2, that $h_{0}$ is a Dirichlet form.

Let $h_{\mathfrak{A} r}$ be the regular part of $\left.h\right|_{\mathfrak{A}}$ as in Sim78. Then $\mathfrak{A}=D\left(h_{\mathfrak{A} r}\right)$. Hence $\mathfrak{A}$ is dense

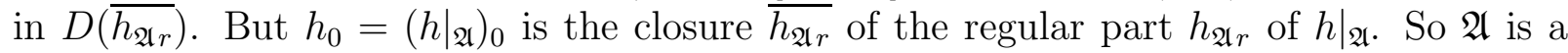
core for $h_{0}$.

Since $h \leq\left.\lambda l\right|_{\mathfrak{A}}$ it follows that $h_{0} \leq \lambda\left(\left.l\right|_{\mathfrak{A})_{0}}=\lambda l\right.$. But by the above $D(l)$ is a core for $h_{0}$. It then easily follows that any core for $l$ is also a core for $h_{0}$.

Finally, since $D(l) \cap C_{c}(X)$ is dense in $C_{0}(X)$ and $D(l) \cap C_{c}(X) \subset D\left(h_{0}\right) \cap C_{c}(X)$ it follows that $D\left(h_{0}\right) \cap C_{c}(X)$ is dense both in $D\left(h_{0}\right)$ and in $C_{0}(X)$.

Next set $h_{\varepsilon}=\tilde{h}+\varepsilon l$ for all $\varepsilon>0$ and $h \in \mathcal{C}_{l}$, where $\tilde{h}$ is the unique form given by Corollary 3.6. Then $D\left(h_{\varepsilon}\right)=D(l)$.

Corollary 3.9 If $h \in \mathcal{C}_{l}$ then $h_{\varepsilon}$ is a diffusion for all $\varepsilon>0$. Moreover, $r . \lim _{\varepsilon \downarrow 0} h_{\varepsilon}=h_{0}$.

Proof If $h \leq\left.\lambda l\right|_{\mathfrak{A}}$ then $\varepsilon l \leq h_{\varepsilon} \leq(\lambda+\varepsilon) l$. Since $l$ is closed it follows that $h_{\varepsilon}$ is closed. Therefore $h_{\varepsilon}=\left(h_{\varepsilon}\right)_{0}$ is a regular Dirichlet form by Lemma 3.8. Moreover, $\mathfrak{A}$ is a core for $h_{\varepsilon}$ and $\left.h_{\varepsilon}\right|_{\mathfrak{A}}$ is strongly local. Therefore $h_{\varepsilon}$ is strongly local by [BH91], Remark I.5.1.5 and Proposition I.5.1.3 $\left(L_{0}\right) \Rightarrow\left(L_{2}\right)$.

If $\tilde{h}$ is the unique form as in Corollary 3.6 then

$$
\underset{\varepsilon \downarrow 0}{\operatorname{rim}} h_{\varepsilon}=(\tilde{h})_{0}=\left(\left.\tilde{h}\right|_{\mathfrak{A}}\right)_{0}=\left(\left.h\right|_{\mathfrak{A}}\right)_{0}=h_{0}
$$

where we used that $\mathfrak{A}$ is dense in $D(\tilde{h})$ and in $D(h)$.

Next we derive a crude $L_{2}$ off-diagonal bound.

If $\mathcal{E}$ is a Dirichlet form over $X$ then we denote by $S=S^{(c e)}$ the semigroup generated by the self-adjoint operator associated with $\mathcal{E}$.

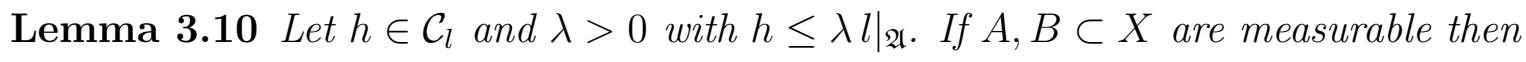

$$
\left|\left(\psi, S_{t}^{\left(h_{0}\right)} \varphi\right)\right| \leq e^{-(4 \lambda t)^{-1} d^{(l)}(A ; B)^{2}}\|\psi\|_{2}\|\varphi\|_{2}
$$

for all $t>0, \varphi \in L_{2}(A)$ and $\psi \in L_{2}(B)$.

Proof For all $\varepsilon>0$ the form $h_{\varepsilon}$ is a diffusion with $h_{\varepsilon} \leq(\lambda+\varepsilon) l$ and $D\left(h_{\varepsilon}\right)=D(l)$ by Corollary [3.9. So

$$
\mathcal{I}_{\psi}^{\left(h_{\varepsilon}\right)}(\varphi) \leq \mathcal{I}_{\psi}^{((\lambda+\varepsilon) l)}(\varphi)=\mathcal{I}_{(\lambda+\varepsilon)^{1 / 2} \psi}^{(l)}(\varphi)
$$


for all $\varphi, \psi \in D(l) \cap L_{\infty}(X)$ with $\varphi \geq 0$ by [ERSZ05], Proposition 3.2. Therefore $\left\|\widehat{\mathcal{I}}_{\psi}^{\left(h_{\varepsilon}\right)}\right\| \mid \leq$ $\left\|\widehat{\mathcal{I}}_{(\lambda+\varepsilon)^{1 / 2} \psi}^{(l)}\right\| \mid$ for all $\psi \in D(l)_{\operatorname{loc}} \cap L_{\infty}(X)=D\left(h_{\varepsilon}\right)_{\operatorname{loc}} \cap L_{\infty}(X)$. So $(\lambda+\varepsilon)^{-1 / 2} d^{(l)}(A ; B) \leq$ $d^{\left(h_{\varepsilon}\right)}(A ; B)$ for all measurable $A, B \subset X$.

The form $h_{\varepsilon}$ is regular by Corollary 3.9. Then it follows from Theorem 2.1, that

$$
\left|\left(\psi, S_{t}^{\left(h_{\varepsilon}\right)} \varphi\right)\right| \leq e^{-(4 t)^{-1} d^{\left(h_{\varepsilon}\right)}(A ; B)^{2}}\|\psi\|_{2}\|\varphi\|_{2} \leq e^{-(4(\lambda+\varepsilon) t)^{-1} d^{(l)}(A ; B)^{2}}\|\psi\|_{2}\|\varphi\|_{2}
$$

for all $t>0$, measurable $A, B \subset X, \varphi \in L_{2}(A)$ and $\psi \in L_{2}(B)$. Since $\lim _{\varepsilon \downarrow 0} S_{t}^{\left(h_{\varepsilon}\right)}=S_{t}^{\left(h_{0}\right)}$ strongly, as a consequence of strong resolvent convergence, the lemma follows.

To exploit the $L_{2}$ off-diagonal bound we need an estimate for $d^{(l)}(A ; B)$. One can obtain a simple estimate if $l$ satisfies Condition L. Therefore from now on we always assume that $l$ satisfies Condition L.

Recall that if $A, B \subset X$ with $A \neq \emptyset$ and $B \neq \emptyset$ then $d^{(e)}(A ; B)=\inf _{x \in A} \inf _{y \in B} d^{(e)}(x ; y)$.

\section{Lemma 3.11}

I. If $U, V \subset X$ are open and non-empty then $d^{(l)}(U ; V) \geq d^{(e)}(U ; V)$.

II. If $K_{1}, K_{2} \subset X$ are compact with $K_{1} \cap K_{2}=\emptyset$ then $d^{(l)}\left(K_{1} ; K_{2}\right)>0$.

Proof The first statement is easy. In order to prove the second one, we use regularity of the metric space $X$. There are non-empty open subsets $U_{1}, U_{2}$ and closed subsets $F_{1}, F_{2}$ such that $K_{1} \subset U_{1} \subset F_{1}, K_{2} \subset U_{2} \subset F_{2}$ and $F_{1} \cap F_{2}=\emptyset$. Then

$$
d^{(l)}\left(K_{1} ; K_{2}\right) \geq d^{(l)}\left(U_{1} ; U_{2}\right) \geq d^{(e)}\left(U_{1} ; U_{2}\right) \geq d^{(e)}\left(F_{1} ; F_{2}\right)>0,
$$

from which the lemma follows.

We next examine local approximations for the Markovian forms $h \in \mathcal{C}_{l}$ defined by truncation. We adopt the definition used for Dirichlet forms and demonstrate that the domination property allows one to deduce many of the standard properties for the relaxations.

If $h \in \mathcal{C}_{l}$ and $\Phi \in \mathfrak{A}$ with $\Phi \geq 0$ then the quadratic form $h_{\Phi}$ is defined by $D\left(h_{\Phi}\right)=\mathfrak{A}$ and

$$
h_{\Phi}(\varphi)=h(\Phi \varphi, \varphi)-2^{-1} h\left(\Phi, \varphi^{2}\right)
$$

for all $\varphi \in \mathfrak{A}$. This is well-defined since $\mathfrak{A}$ is an algebra. Moreover, $h_{\Phi}(\varphi)=\mathcal{I}_{\varphi}^{(h)}(\Phi)$ if $h$ is a Dirichlet form. Now the truncation $h_{\varepsilon \Phi}$ of the Dirichlet form $h_{\varepsilon}$ satisfies

$$
0 \leq h_{\varepsilon \Phi}(\varphi)=\mathcal{I}_{\varphi}^{\left(h_{\varepsilon}\right)}(\Phi) \leq\|\Phi\|_{\infty} h_{\varepsilon}(\varphi)=\|\Phi\|_{\infty}(h(\varphi)+\varepsilon l(\varphi))
$$

for all $\varphi \in \mathfrak{A}$ and $\varepsilon>0$, where we have used (4). But $h_{\varepsilon \Phi}(\varphi)=h_{\Phi}(\varphi)+\varepsilon l_{\Phi}(\varphi)$. Hence in the limit $\varepsilon \downarrow 0$ one deduces that

$$
0 \leq h_{\Phi}(\varphi) \leq\|\Phi\|_{\infty} h(\varphi) \leq \lambda\|\Phi\|_{\infty} l(\varphi)
$$

for all $\varphi \in \mathfrak{A}$. One deduces in a similar fashion that $h_{\Phi}$ satisfies the basic Markov property: $\varphi \in \mathfrak{A}$ implies $0 \vee \varphi \wedge \mathbb{1} \in \mathfrak{A}$ and $h_{\Phi}(0 \vee \varphi \wedge \mathbb{1}) \leq h_{\Phi}(\varphi)$. Moreover, $h_{\Phi}$ is strongly local. Hence $h_{\Phi} \in \mathcal{C}_{l}$.

Note that in general $h_{\Phi}$ is not a Dirichlet form as it is not necessarily closed and possibly not closable, even if $h$ is a Dirichlet form. The relaxation, or viscosity closure, $h_{\Phi 0}=\left(h_{\Phi}\right)_{0}$ is, however, a regular Dirichlet form by Lemma 3.8 and the next lemma establishes that it is even a diffusion if $\operatorname{supp} \Phi$ is compact. 
Lemma 3.12 Let $h \in \mathcal{C}_{l}$ and $\Phi \in \mathfrak{A}$ with $0 \leq \Phi \leq 1$ and $\operatorname{supp} \Phi$ compact. Then $h_{\Phi 0} \in \mathcal{C}_{l}$ and $h_{\Phi 0}$ is a diffusion.

Moreover, $S^{\left(h_{\Phi 0}\right)}$ leaves $L_{2}(\operatorname{supp} \Phi)$ invariant and $S_{t}^{\left(h_{\Phi 0}\right)} \varphi=\varphi$ for all $\varphi \in L_{2}\left((\operatorname{supp} \Phi)^{\mathrm{c}}\right)$ and $t>0$.

Proof For brevity write $S=S^{\left(h_{\Phi 0}\right)}$.

It follows from the foregoing that $h_{\Phi} \in \mathcal{C}_{l}$. Therefore $h_{\Phi 0}$ is a regular Dirichlet form by the first statement of Lemma 3.8 and $D(l)$ is dense in $D\left(h_{\Phi 0}\right)$ by the second statement of the lemma. Next we establish the localization properties of $h_{\Phi 0}$ and the semigroup $S$.

Since $l$ is regular there are $\chi_{1}, \chi_{2}, \ldots \in D(l) \cap C_{c}(X)$ such that $\lim _{n \rightarrow \infty} \chi_{n}=\mathbb{1}_{\text {supp } \Phi}$ in $L_{2}(X)$ and for all $n \in \mathbf{N}$ there is a neighbourhood $U$ of $\operatorname{supp} \Phi$ with $\left.\chi_{n}\right|_{U}=1$. Then

$$
h_{\Phi}\left(\chi_{n}\right)=h\left(\Phi, \chi_{n}\right)-2^{-1} h\left(\Phi, \chi_{n}^{2}\right)=0
$$

because $h$ is strongly local. Moreover

$$
h_{\Phi 0}\left(\mathbb{1}_{\text {supp } \Phi}\right) \leq \liminf _{n \rightarrow \infty} h_{\Phi}\left(\chi_{n}\right)=0
$$

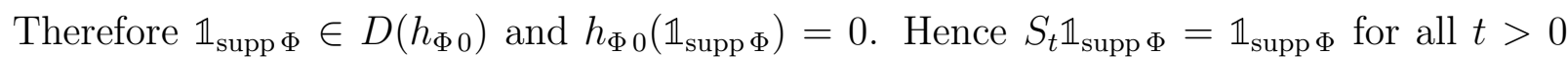
and $S$ leaves $L_{2}(\operatorname{supp} \Phi)$ invariant. (Cf. ERSZ04 Lemma 6.1.) Similarly, if $\varphi \in \mathfrak{A}$ then $h_{\varepsilon \Phi}\left(\left(\mathbb{1}-\chi_{n}\right) \varphi\right)=-2^{-1} h_{\varepsilon}\left(\Phi,\left(1-\chi_{n}\right)^{2} \varphi^{2}\right)=0$ for all $n \in \mathbf{N}$ and $\varepsilon>0$ by [BH]-locality of $h_{\varepsilon}$. So $h_{\Phi}\left(\left(\mathbb{1}-\chi_{n}\right) \varphi\right)=\lim _{\varepsilon \downarrow 0} h_{\varepsilon \Phi}\left(\left(\mathbb{1}-\chi_{n}\right) \varphi\right)=0$ and

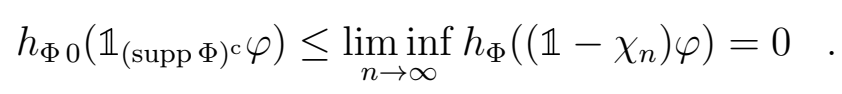

Now let $\varphi \in L_{2}\left((\operatorname{supp} \Phi)^{\mathrm{c}}\right)$. Since $\mathfrak{A}$ is dense in $L_{2}(X)$ there are $\varphi_{1}, \varphi_{2}, \ldots \in \mathfrak{A}$ such that $\lim _{n \rightarrow \infty} \varphi_{n}=\varphi$ in $L_{2}(X)$. Then $\lim _{n \rightarrow \infty} \mathbb{1}_{(\operatorname{supp} \Phi)^{\mathrm{c}}} \varphi_{n}=\varphi$ in $L_{2}(X)$. Since $h_{\Phi 0}$ is lower semicontinuous one deduces that $h_{\Phi 0}(\varphi) \leq \liminf _{n \rightarrow \infty} h_{\Phi 0}\left(\mathbb{1}_{(\operatorname{supp} \Phi)^{\mathrm{c}}} \varphi\right)=0$. So $\varphi \in D\left(h_{\Phi 0}\right)$ and $h_{\Phi 0}(\varphi)=0$. Hence $S_{t}^{\left(h_{\Phi 0}\right)} \varphi=\varphi$ for all $\varphi \in L_{2}\left((\operatorname{supp} \Phi)^{\mathrm{c}}\right)$ and $t>0$.

Finally we prove that $h_{\Phi 0}$ is strongly local. First, if $h_{\Phi} \leq\left.\lambda l\right|_{\mathfrak{A}}$ with $\lambda>0$ then

$$
\left|\left(\psi, S_{t} \varphi\right)\right| \leq e^{-(4 \mu t)^{-1} d^{(l)}(A ; B)^{2}}\|\psi\|_{2}\|\varphi\|_{2}
$$

for all measurable $A, B \subset X, t>0, \varphi \in L_{2}(A)$ and $\psi \in L_{2}(B)$ by Lemma 3.10] and (8), where $\mu=\lambda\|\Phi\|_{\infty}+1$. Secondly, let $\varphi, \psi \in D\left(h_{\Phi 0}\right)$ with $\operatorname{supp} \varphi$ and $\operatorname{supp} \psi \operatorname{compact}$ and suppose there exists a neighbourhood $U$ of $\operatorname{supp} \varphi$ such that $\left.\psi\right|_{U}=1$. There exists a $\chi \in D(l) \cap C_{c}(X)$ such that $K=\operatorname{supp} \chi$ is compact and $\left.\chi\right|_{\operatorname{supp} \psi \cup \operatorname{supp} \Phi}=1$. Then

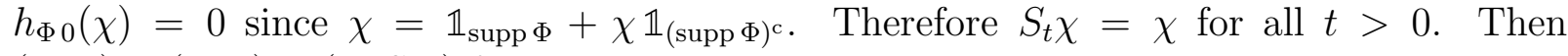
$(\psi, \varphi)=(\chi, \varphi)=\left(\chi, S_{t} \varphi\right)$ for all $t>0$. Hence

$$
t^{-1}\left|\left(\psi,\left(I-S_{t}\right) \varphi\right)\right|=t^{-1}\left|\left(\chi-\psi, S_{t} \varphi\right)\right| \leq t^{-1} e^{-(4 \mu t)^{-1} d^{(l)}(K \backslash U ; \operatorname{supp} \varphi)^{2}}\|\chi-\psi\|_{2}\|\varphi\|_{2}
$$

for all $t>0$. But $d^{(l)}(K \backslash U ; \operatorname{supp} \varphi)>0$ by Condition L and Lemma 3.11. So

$$
\left|h_{\Phi 0}(\psi, \varphi)\right|=\lim _{t \downarrow 0} t^{-1}\left|\left(\psi,\left(I-S_{t}\right) \varphi\right)\right|=0
$$

Thus $h_{\Phi 0}$ is strongly local. This completes the verification that $h_{\Phi 0}$ is a diffusion and $h_{\Phi 0} \in \mathcal{C}_{l}$. 
If $h \in \mathcal{C}_{l}$ and $\Phi \in \mathfrak{A}$ with $\operatorname{supp} \Phi$ compact and $0 \leq \Phi \leq 1$ write $\mathcal{I}^{(\Phi)}=\mathcal{I}^{\left(h_{\Phi 0}\right)}$ and $d^{(\Phi)}=d^{\left(h_{\Phi 0}\right)}$. Further let $H_{\Phi 0}$ and $S^{(\Phi)}$ denote the operator and semigroup associated with the form $h_{\Phi 0}$. Moreover, let $H_{0}$ denote the operator associated with the form $h_{0}$. It will be clear from the context which $h$ is involved. Proposition 3.12 establishes that $h_{\Phi 0}$ is a localization of $h$ : the corresponding semigroup $S^{\Phi}$ leaves $L_{2}(\operatorname{supp} \Phi)$ invariant. Next we consider the distance corresponding to the generator of the restriction of $S^{\Phi}$ to $L_{2}(\operatorname{supp} \Phi)$ and its relation to the distance corresponding to $h_{\Phi 0}$.

Lemma 3.13 Let $h \in \mathcal{C}_{l}$ and $\Phi \in \mathfrak{A}$ with $0 \leq \Phi \leq 1$ and supp $\Phi$ compact. Define the form $\hat{h}$ on $L_{2}(\operatorname{supp} \Phi)$ by

$$
D(\hat{h})=\left\{\left.\varphi\right|_{\operatorname{supp} \Phi}: \varphi \in D\left(h_{\Phi 0}\right)\right\}
$$

and

$$
\hat{h}\left(\left.\varphi\right|_{\operatorname{supp} \Phi}\right)=h_{\Phi 0}\left(\varphi \mathbb{1}_{\operatorname{supp} \Phi}\right)
$$

for all $\varphi \in D\left(h_{\Phi 0}\right)$. Then

$$
d^{(\Phi)}(A ; B)=d^{(\hat{h})}(A \cap \operatorname{supp} \Phi ; B \cap \operatorname{supp} \Phi)
$$

for all measurable $A, B \subset X$.

Proof Note that the form $\hat{h}$ is a conservative [BH]-local Dirichlet form. Moreover, $D(\hat{h})_{\text {loc }}=D(\hat{h})$. If $\varphi, \psi \in D\left(h_{\Phi 0}\right) \cap L_{\infty}$ then

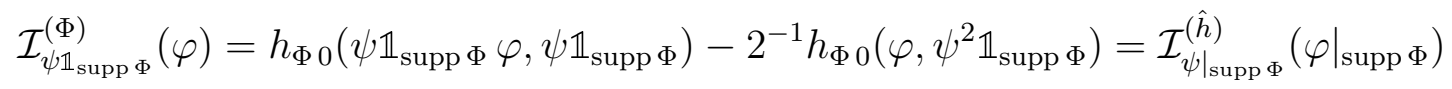

since $h_{\Phi 0}\left(\mathbb{1}_{(\operatorname{supp} \Phi)^{\mathrm{c}}} \varphi\right)=0$. So $\left\|\left|\widehat{\mathcal{I}}_{\psi \mathbb{1}_{\text {supp } \Phi}}^{(\Phi)}\left\|\left|=\left\|\left|\widehat{\mathcal{I}}_{\left.\psi\right|_{\text {supp } \Phi} ^{(\hat{h})}}\right|\right\|\right.\right.\right.\right.$ for all $\psi \in D\left(h_{\Phi 0}\right)_{\text {loc }} \cap L_{\infty}$.

Let $M \in\left[0, d^{(\Phi)}(A ; B)\right] \cap \mathbf{R}$ and $\varepsilon>0$. By [ERSZ05], Lemma 4.2.III, there exists a $\psi \in D_{0}\left(h_{\Phi 0}\right)$ such that $\left.\psi\right|_{B}=0$ and $\left.\psi\right|_{A} \geq M-\varepsilon$. Then $\left.\psi\right|_{\operatorname{supp} \Phi} \in D_{0}(\hat{h})$. Therefore

$$
M-\varepsilon \leq d_{\psi}(A ; B) \leq d_{\left.\psi\right|_{\text {supp } \Phi}}(A \cap \operatorname{supp} \Phi ; B \cap \operatorname{supp} \Phi) \leq d^{(\hat{h})}(A \cap \operatorname{supp} \Phi ; B \cap \operatorname{supp} \Phi) .
$$

So $d^{(\Phi)}(A ; B) \leq d^{(\hat{h})}(A \cap \operatorname{supp} \Phi ; B \cap \operatorname{supp} \Phi)$.

Conversely, let $M \in\left[0, d^{(\hat{h})}(A \cap \operatorname{supp} \Phi ; B \cap \operatorname{supp} \Phi)\right] \cap \mathbf{R}$ and $\varepsilon>0$. By another application of Lemma 4.2.III in [ERSZ05] there exists a $\tilde{\psi} \in D_{0}(\hat{h})$ such that $\left.\tilde{\psi}\right|_{B \cap \operatorname{supp} \Phi}=0$ and $\left.\tilde{\psi}\right|_{A \cap \operatorname{supp} \Phi} \geq M-\varepsilon$. There exists a $\psi \in D\left(h_{\Phi 0}\right)$ such that $\left.\psi\right|_{\operatorname{supp} \Phi}=\tilde{\psi}$. Then $\psi \mathbb{1}_{\text {supp } \Phi} \in D_{0}\left(h_{\Phi 0}\right)$. Moreover, $\widehat{\mathcal{I}}_{\mathbb{1}_{(\operatorname{supp} \Phi)^{\mathrm{c}} \cap A}^{(\Phi)}}=0$ by the last part of Lemma 3.12 . Let $\tau=(M-\varepsilon) \mathbb{1}_{(\operatorname{supp} \Phi)^{\mathrm{c}} \cap A}+\psi \mathbb{1}_{\text {supp } \Phi}$. Then $\tau \in D_{0}\left(h_{\Phi 0}\right)_{\text {loc }}$ by [ERSZ05], Lemma 3.3.I, $\left.\tau\right|_{B}=0$ and $\left.\tau\right|_{A} \geq M-\varepsilon$. Therefore

$$
M-\varepsilon \leq d_{\tau}(A ; B) \leq d^{(\Phi)}(A ; B)
$$

and $d^{(\hat{h})}(A \cap \operatorname{supp} \Phi ; B \cap \operatorname{supp} \Phi) \leq d^{(\Phi)}(A ; B)$.

Now we can make the first key deduction in the proof of the theorem. One can apply the results of Hino-Ramírez [HR03] to the form $\hat{h}$ in Lemma 3.13.

Corollary 3.14 Let $h \in \mathcal{C}_{l}$ and $\Phi \in \mathfrak{A}$ with $0 \leq \Phi \leq 1$ and $\operatorname{supp} \Phi$ compact. Let $A, B \subset X$ be measurable with $A, B \subseteq \operatorname{supp} \Phi$. Then

$$
\lim _{t \downarrow 0} t \log \left(\mathbb{1}_{A}, S_{t}^{(\Phi)} \mathbb{1}_{B}\right)=-4^{-1} d^{(\Phi)}(A ; B)^{2} .
$$


Proof This follows from Lemma 3.13 and HR03, Theorem 1.1.

The next idea is to take a limit over the localizations $\Phi$ as $\Phi$ increases monotonically to the identity function. This involves analyzing both the limit of the semigroups $S^{(\Phi)}$ and of the distances $d^{(\Phi)}$. It is not difficult using arguments of monotonicity to deduce that the strong limit of the semigroups $S^{(\Phi)}$ and the pointwise limit of the distances $d^{(\Phi)}$ exist. But we also have to identify the limits with the semigroup and distance corresponding to the relaxation and to control simultaneously the small time behaviour. The key to this analysis is the observation that the associated wave equations have a finite speed of propagation (see ERSZ04, Proposition 3.2).

If $A \subset X$ with $A \neq \emptyset$ and $r>0$ set

$$
\widetilde{B}^{(e)}(A ; r)=\left\{x \in X: d^{(e)}(x ; A) \leq r\right\},
$$

where $d^{(e)}(x ; A)=\inf \{|x-a|: a \in A\}$. Note that $\widetilde{B}^{(e)}(A ; r)$ is closed since $x \mapsto d^{(e)}(x ; A)$ is continuous. Since $h_{\Phi} \leq\left.\|\Phi\|_{\infty} h\right|_{\mathfrak{A}}$ by (18) one has $h_{\Phi 0} \leq\|\Phi\|_{\infty}\left(\left.h\right|_{\mathfrak{A}}\right)_{0}=\|\Phi\|_{\infty} h_{0}$, so clearly $D\left(h_{0}\right) \subset D\left(h_{\Phi 0}\right)$ if $\Phi \in D(l) \cap L_{\infty}$ with $\Phi \geq 0$.

Proposition 3.15 Let $h \in \mathcal{C}_{l}, \lambda>0$ and $\Phi \in \mathfrak{A}$ and suppose that $h \leq\left.\lambda l\right|_{\mathfrak{A}}, 0 \leq \Phi \leq 1$ and supp $\Phi$ is compact. Further let $A \subset X$ measurable, $\Omega \subset X$ open with $\emptyset \neq \bar{A} \subset \Omega$ and suppose $\left.\Phi\right|_{\Omega}=1$. Then

$$
\cos \left(t H_{0}^{1 / 2}\right) \varphi=\cos \left(t H_{\Phi 0}^{1 / 2}\right) \varphi
$$

for all $t \in \mathbf{R}$ with $|t| \leq \lambda^{-1 / 2} d^{(e)}\left(A ; \Omega^{\mathrm{c}}\right)$ and $\varphi \in L_{2}(A)$. Moreover, if $\psi \in D\left(h_{\Phi 0}\right)$ and $\operatorname{supp} \psi \subset \Omega$ then $\psi \in D\left(h_{0}\right)$ and $h_{0}(\psi)=h_{\Phi 0}(\psi)$.

The proof of the proposition relies on two lemmas.

Lemma 3.16 Let $h \in \mathcal{C}_{l}$ be a diffusion, $\lambda>0$ with $h \leq\left.\lambda l\right|_{\mathfrak{A}}$, and let $H$ denote the corresponding positive self-adjoint operator. If $A \subset X$ is open with $A \neq \emptyset$ then

$$
\cos \left(t H^{1 / 2}\right) L_{2}(A) \subset L_{2}\left(\widetilde{B}^{(e)}\left(A ; \lambda^{1 / 2}|t|\right)\right)
$$

and

$$
\left(t H^{1 / 2}\right)^{-1} \sin \left(t H^{1 / 2}\right) L_{2}(A) \subset L_{2}\left(\widetilde{B}^{(e)}\left(A ; \lambda^{1 / 2}|t|\right)\right)
$$

for all $t \in \mathbf{R} \backslash\{0\}$.

Here and in the sequel the operator formally denoted by $\left(t H^{1 / 2}\right)^{-1} \sin \left(t H^{1 / 2}\right)$ is properly defined by spectral theory, even if $H=0$.

Proof It follows from Lemmas 3.10 and 3.11] that

$$
\left|\left(\psi, S_{t}^{(h)} \varphi\right)\right| \leq e^{-(4 \lambda t)^{-1} d^{(e)}(A ; B)^{2}}\|\psi\|_{2}\|\varphi\|_{2}
$$

for all $t>0$, non-empty open $B \subset X, \varphi \in L_{2}(A)$ and $\psi \in L_{2}(B)$. Therefore

$$
\left(\psi, \cos \left(t H^{1 / 2}\right) \varphi\right)=0
$$

for all non-empty open $B \subset X, \varphi \in L_{2}(A), \psi \in L_{2}(B)$ and $t \in \mathbf{R}$ with $|t| \leq \lambda^{-1 / 2} d^{(e)}(A ; B)$ by ERSZ04, Lemma 3.3. Hence if $r>0, \psi \in C_{c}\left(\widetilde{B}^{(e)}(A ; r)^{\mathrm{c}}\right)$ and $\varphi \in L_{2}(A)$ then 
$\left(\psi, \cos \left(t H^{1 / 2}\right) \varphi\right)=0$ for all $|t| \leq \lambda^{-1 / 2} r$. This implies the first identity. The second identity follows from the observation that

$$
\left(\psi,\left(t H^{1 / 2}\right)^{-1} \sin \left(t H^{1 / 2}\right) \varphi\right)=t^{-1} \int_{0}^{t} d s\left(\psi, \cos \left(s H^{1 / 2}\right) \varphi\right)
$$

combined with the first identity.

Lemma 3.17 Let $h \in \mathcal{C}_{l}$ be a diffusion and let $H$ denote the corresponding positive selfadjoint operator. If $A \subset X$ is open then $\overline{D(H) \cap L_{2}(A)}=L_{2}(A)$.

Proof We may assume that $A \neq \emptyset$. Let $\lambda>0$ and suppose that $h \leq\left.\lambda l\right|_{\mathfrak{A} \text {. Let }}$ $\varphi \in C_{c}(A)$ with $\varphi \neq 0$. For all $t>0$ set $\varphi_{t}=\left(t^{2} H\right)^{-1} \sin ^{2}\left(t H^{1 / 2}\right) \varphi$. Then $\varphi_{t} \in D(H)$ and $\varphi_{t} \in L_{2}\left(\widetilde{B}^{(e)}\left(\operatorname{supp} \varphi ; 2 \lambda^{1 / 2}|t|\right)\right)$ for all $t>0$ by Lemma 3.16. Hence $\varphi_{t} \in L_{2}(A)$ if $t>0$ is small enough. Finally, $\lim _{t \downarrow 0} \varphi_{t}=\varphi$ by spectral theory. So $C_{c}(A) \subset \overline{D(H) \cap L_{2}(A)}$ and the lemma follows.

The conclusion of the last lemma is very useful since it shows that the operator domain contains abundant functions with compact support.

Proof of Proposition [3.15] Fix $\varepsilon>0$. We begin by comparing the actions of the operators $H_{\varepsilon}$ and $H_{\Phi \varepsilon}$ corresponding to the diffusions $h_{\varepsilon}$ and $h_{\Phi \varepsilon}$ associated with $h$ and $h_{\Phi}$ (see Corollary [3.9).

First assume that $A$ is an open subset of $X$ and let $r \in\left\langle 0,(\lambda+\varepsilon)^{-1 / 2} d^{(e)}\left(A ; \Omega^{\mathrm{c}}\right)\right\rangle$. Fix $\varphi \in L_{2}(A)$. Then it follows from Lemma 3.16 applied to $h_{\varepsilon}$ that

$$
\cos \left(t H_{\varepsilon}^{1 / 2}\right) \varphi \in L_{2}\left(\widetilde{B}^{(e)}\left(A ;(\lambda+\varepsilon)^{1 / 2}|t|\right)\right) \subset L_{2}\left(\widetilde{B}^{(e)}\left(A ;(\lambda+\varepsilon)^{1 / 2} r\right)\right) \subset L_{2}(\Omega)
$$

for all $t \in\langle-r, r\rangle$.

If in addition $\varphi \in D\left(h_{\varepsilon}\right)=D(l)$ then $\cos \left(t H_{\varepsilon}^{1 / 2}\right) \varphi \in D(l)$. Thus if one sets $\varphi_{n}=$ $(-n) \vee \cos \left(t H_{\varepsilon}^{1 / 2}\right) \varphi \wedge n$ for all $n \in \mathbf{N}$ one has $\varphi_{n} \in D(l) \cap L_{\infty}(X)=\mathfrak{A}$ and $\operatorname{supp} \varphi_{n} \subset$ $\widetilde{B}^{(e)}\left(A ;(\lambda+\varepsilon)^{1 / 2} r\right)$. Since $l$ is regular there exists a $\chi \in \mathfrak{A}$ with $0 \leq \chi \leq 1$, supp $\chi \subset \Omega$ and $\left.\chi\right|_{\widetilde{B}^{(e)}\left(A ;(\lambda+\varepsilon)^{1 / 2} r\right)}=1$. Let $\psi \in \mathfrak{A}$. Then $\chi \psi \in \mathfrak{A}$ and $h_{\Phi \varepsilon}\left(\psi, \varphi_{n}\right)=h_{\Phi \varepsilon}\left(\chi \psi, \varphi_{n}\right)$ by locality. But

$$
\begin{aligned}
h_{\Phi \varepsilon}\left(\chi \psi, \varphi_{n}\right) & =h_{\Phi}\left(\chi \psi, \varphi_{n}\right)+\varepsilon l\left(\chi \psi, \varphi_{n}\right) \\
& =h\left(\chi \psi, \varphi_{n}\right)+\varepsilon l\left(\chi \psi, \varphi_{n}\right)=h_{\varepsilon}\left(\chi \psi, \varphi_{n}\right)
\end{aligned}
$$

by the definition of $h_{\Phi}$ and the assumption $\left.\Phi\right|_{\Omega}=1$. Therefore

$$
h_{\Phi \varepsilon}\left(\psi, \varphi_{n}\right)=h_{\varepsilon}\left(\chi \psi, \varphi_{n}\right)=h_{\varepsilon}\left(\psi, \varphi_{n}\right)
$$

where the second identity follows by another use of locality. Then the limit $n \rightarrow \infty$ gives

$$
h_{\Phi \varepsilon}\left(\psi, \cos \left(t H_{\varepsilon}^{1 / 2}\right) \varphi\right)=h_{\varepsilon}\left(\psi, \cos \left(t H_{\varepsilon}^{1 / 2}\right) \varphi\right)
$$

for all $\psi \in \mathfrak{A}, \varphi \in D\left(h_{\varepsilon}\right) \cap L_{2}(A)$ and $t \in\langle-r, r\rangle$ by [FOT94], Theorem 1.4.2(iii).

Let $\varphi \in D\left(H_{\varepsilon}\right) \cap L_{2}(A)$ and $t \in\langle-r, r\rangle$. Because $\mathfrak{A}$ is a core for $h_{\Phi \varepsilon}$ one has

$$
h_{\Phi \varepsilon}\left(\psi, \cos \left(t H_{\varepsilon}^{1 / 2}\right) \varphi\right)=h_{\varepsilon}\left(\psi, \cos \left(t H_{\varepsilon}^{1 / 2}\right) \varphi\right)=\left(\psi, \cos \left(t H_{\varepsilon}^{1 / 2}\right) H_{\varepsilon} \varphi\right)
$$


for all $\psi \in D\left(h_{\Phi \varepsilon}\right)$. Since the form $h_{\Phi \varepsilon}$ is closed it follows that $\cos \left(t H_{\varepsilon}^{1 / 2}\right) \varphi \in D\left(H_{\Phi \varepsilon}\right)$ and

$$
H_{\Phi \varepsilon} \cos \left(t H_{\varepsilon}^{1 / 2}\right) \varphi=\cos \left(t H_{\varepsilon}^{1 / 2}\right) H_{\varepsilon} \varphi
$$

for all $t \in\langle-r, r\rangle$ and $\varphi \in D\left(H_{\varepsilon}\right) \cap L_{2}(A)$.

Next let $\varphi \in D\left(H_{\varepsilon}\right) \cap L_{2}(A)$ and for all $t \in \mathbf{R}$ define

$$
\chi_{t}=\cos \left(t H_{\varepsilon}^{1 / 2}\right) \varphi-\cos \left(t H_{\Phi \varepsilon}^{1 / 2}\right) \varphi .
$$

Clearly $\left\|\chi_{t}\right\|_{2} \leq 2\|\varphi\|_{2}$ for all $t \in \mathbf{R}$. Let $\psi \in D\left(H_{\Phi \varepsilon}\right)$. Then $\tau: t \mapsto\left(\psi, \chi_{t}\right)$ is twice differentiable and $\tau(0)=\tau^{\prime}(0)=0$. Moreover,

$$
\begin{aligned}
\left(\psi, \chi_{t}\right) & =\int_{0}^{t} d t_{1} \int_{0}^{t_{1}} d t_{2} \tau^{\prime \prime}\left(t_{2}\right) \\
& =\int_{0}^{t} d t_{1} \int_{0}^{t_{1}} d t_{2}\left(\left(H_{\Phi \varepsilon} \psi, \cos \left(t_{2} H_{\Phi \varepsilon}^{1 / 2}\right) \varphi\right)-\left(\psi, \cos \left(t_{2} H_{\varepsilon}^{1 / 2}\right) H_{\varepsilon} \varphi\right)\right) \\
& =-\int_{0}^{t} d t_{1} \int_{0}^{t_{1}} d t_{2}\left(H_{\Phi \varepsilon} \psi, \chi_{t_{2}}\right)
\end{aligned}
$$

for all $t \in\langle-r, r\rangle$, where we used (10) in the last step. Now assume that $\psi$ is a bounded vector for $H_{\Phi \varepsilon}$, i.e., $\psi \in \bigcap_{n=1}^{\infty} D\left(H_{\Phi \varepsilon}^{n}\right)$ and there exists a $b>0$ such that $\left\|H_{\Phi \varepsilon}^{n} \psi\right\|_{2} \leq b^{n}$ for all $n \in \mathbf{N}$. Then by iteration one deduces that

$$
\begin{aligned}
\left|\left(\psi, \chi_{t}\right)\right| & =\left|\int_{0}^{t} d t_{1} \ldots \int_{0}^{t_{2 n-1}} d t_{2 n}\left(H_{\Phi \varepsilon}^{n} \psi, \chi_{t_{2 n}}\right)\right| \\
& \leq 2|t|^{2 n}(2 n) !^{-1}\left\|H_{\Phi \varepsilon}^{n} \psi\right\|_{2}\|\varphi\|_{2} \leq 2|t|^{2 n}(2 n) !^{-1} b^{n}\|\varphi\|_{2}
\end{aligned}
$$

for all $n \in \mathbf{N}$ and $t \in\langle-r, r\rangle$. Taking the limit $n \rightarrow \infty$ it follows that $\left(\psi, \chi_{t}\right)=0$ for all bounded vectors $\psi$ for $H_{\Phi \varepsilon}$ and $t \in\langle-r, r\rangle$. Since the bounded vectors for $H_{\Phi \varepsilon}$ are dense in $L_{2}$ by spectral theory one deduces that $\cos \left(t H_{\Phi \varepsilon}^{1 / 2}\right) \varphi=\cos \left(t H_{\varepsilon}^{1 / 2}\right) \varphi$ for all $t \in\langle-r, r\rangle$ and $\varphi \in D\left(H_{\varepsilon}\right) \cap L_{2}(A)$. Hence

$$
\cos \left(t H_{\Phi \varepsilon}^{1 / 2}\right) \varphi=\cos \left(t H_{\varepsilon}^{1 / 2}\right) \varphi
$$

for all $t \in\langle-r, r\rangle$ and $\varphi \in L_{2}(A)$ by Lemma 3.17 since $A$ is open.

Let $\varphi \in L_{2}(A)$. Then $\cos \left(t H_{\varepsilon}^{1 / 2}\right) \varphi=\cos \left(t H_{\Phi \varepsilon}^{1 / 2}\right) \varphi$ for all $\varepsilon>0$ and for all $t$ with $|t|<(\lambda+\varepsilon)^{-1 / 2} d^{(e)}\left(A ; \Omega^{\mathrm{c}}\right)$. Therefore

$$
\cos \left(t H_{0}^{1 / 2}\right) \varphi=\lim _{\varepsilon \downarrow 0} \cos \left(t H_{\varepsilon}^{1 / 2}\right) \varphi=\lim _{\varepsilon \downarrow 0} \cos \left(t H_{\Phi \varepsilon}^{1 / 2}\right) \varphi=\cos \left(t H_{\Phi 0}^{1 / 2}\right) \varphi
$$

for all $|t|<\lambda^{-1 / 2} d^{(e)}\left(A ; \Omega^{\mathrm{c}}\right)$ by strong resolvent convergence of $H_{\varepsilon}$ to $H_{0}$ and of $H_{\Phi \varepsilon}$ to $H_{\Phi 0}$ (see [RS72, Theorem VIII.20 and Corollary [3.9). Then the first statement of the proposition follows if $A$ is open.

If $A$ is not open then for all $\delta \in\left\langle 0, d^{(e)}\left(A ; \Omega^{\mathrm{c}}\right)\right\rangle$ one can apply the above to the open set $B^{(e)}(A ; \delta)=\left\{x \in X: d^{(e)}(x ; A)<\delta\right\}$ and deduce that (9) is valid for all $\varphi \in L_{2}(A)$ and $|t|<\lambda^{-1 / 2} d^{(e)}\left(B^{(e)}(A ; \delta) ; \Omega^{\mathrm{c}}\right)$. Since $\lim _{\delta \downarrow 0} d^{(e)}\left(B^{(e)}(A ; \delta) ; \Omega^{\mathrm{c}}\right)=d^{(e)}\left(A ; \Omega^{\mathrm{c}}\right)$ by the triangle inequality the first statement of the proposition follows for general $A$. 
The last statement of the proposition follows since

$$
D\left(h_{0}\right)=\left\{\varphi \in L_{2}(X): \sup _{t \in\langle 0,1]} 2 t^{-2}\left(\varphi,\left(I-\cos \left(t H_{0}^{1 / 2}\right)\right) \varphi\right)<\infty\right\}
$$

and $h_{0}(\varphi)=\lim _{t \downarrow 0} 2 t^{-2}\left(\varphi,\left(I-\cos \left(t H_{0}^{1 / 2}\right)\right) \varphi\right)$ for all $\varphi \in D\left(h_{0}\right)$, with a similar expression for $h_{\Phi 0}$.

The proposition has three very useful corollaries. The first corollary establishes the first statement of Theorem 2.2 .

Corollary 3.18 If $h \in \mathcal{C}_{l}$ then $h_{0}$ is a diffusion. In particular, $h_{0} \in \mathcal{C}_{l}$.

Proof By Lemma 3.8 it remains to show that $h_{0}$ is strongly local. Let $\varphi, \psi \in D\left(h_{0}\right)$ with $\operatorname{supp} \varphi$ and $\operatorname{supp} \psi$ compact and $\psi$ is constant on a neighbourhood of $\operatorname{supp} \varphi$. Since $l$ is regular there exist $\Phi \in \mathfrak{A}$ and an open $\Omega \subset X \operatorname{such}$ that $\operatorname{supp}(\varphi \pm \psi) \subset \Omega, 0 \leq \Phi \leq 1$, $\operatorname{supp} \Phi$ is compact and $\left.\Phi\right|_{\Omega}=1$. Then $h_{\Phi 0}(\psi, \varphi)=0$ by Lemma 3.12. But $h_{0}(\varphi \pm \psi)=$ $h_{\Phi 0}(\varphi \pm \psi)$ by the last part of Proposition 3.15. Therefore $h_{0}(\psi, \varphi)=h_{\Phi 0}(\psi, \varphi)=0$ by polarization and symmetry.

Corollary 3.19 Adopt the assumptions of Proposition 3.15. If $\psi \in D\left(h_{\Phi 0}\right) \cap L_{\infty}$ with $\operatorname{supp} \psi \subset \Omega$ then $\left\|\widehat{\mathcal{I}}_{\psi}^{\left(h_{0}\right)}\right\|\|=\| \mid \widehat{\mathcal{I}}_{\psi}^{(\Phi)}\|\|$.

Proof Since $l$ is regular there exists a $\tau \in D(l) \cap L_{\infty}$ such that $0 \leq \tau \leq 1,\left.\tau\right|_{\operatorname{supp} \psi}=1$ and $\operatorname{supp} \tau \subset \Omega$. Let $\varphi \in D\left(h_{\Phi 0}\right)_{\text {loc }} \cap L_{\infty, c}$. Then $\varphi \tau \in D\left(h_{\Phi 0}\right) \cap L_{\infty}$ and $\varphi \tau \in D\left(h_{0}\right)$ by the last part of Proposition 3.15. Hence by strong locality and Proposition [3.15] one has

$$
\begin{aligned}
\widehat{\mathcal{I}}_{\psi}^{(\Phi)}(\varphi)=\mathcal{I}_{\psi}^{(\Phi)}(\varphi \tau) & =h_{\Phi 0}(\psi \varphi \tau, \psi)-2^{-1} h_{\Phi 0}\left(\varphi \tau, \psi^{2}\right) \\
& =h_{0}(\psi \varphi \tau, \psi)-2^{-1} h_{0}\left(\varphi \tau, \psi^{2}\right)=\widehat{\mathcal{I}}_{\psi}^{\left(h_{0}\right)}(\varphi \tau)
\end{aligned}
$$

So

$$
\left|\widehat{\mathcal{I}}_{\psi}^{(\Phi)}(\varphi)\right| \leq\left\|\left|\widehat{\mathcal{I}}_{\psi}^{\left(h_{0}\right)}\|\|\|\varphi \tau\|_{1} \leq\left\|\mid \widehat{\mathcal{I}}_{\psi}^{\left(h_{0}\right)}\right\|\|\| \varphi \|_{1}\right.\right.
$$

and $\left\|\mid \widehat{\mathcal{I}}_{\psi}^{(\Phi)}\right\|\|\leq\| \widehat{\mathcal{I}}_{\psi}^{\left(h_{0}\right)}\|\|$. The opposite inequality is similar.

The third corollary establishes that the semigroups $S^{(\Phi)}$ converge strongly to the semigroup associated with the relaxation $h_{0}$.

Corollary 3.20 Adopt the assumptions of Proposition 3.15. Then

$$
\left\|S_{t}^{\left(h_{0}\right)} \varphi-S_{t}^{(\Phi)} \varphi\right\|_{2} \leq 2 e^{-(4 t)^{-1} r^{2}}\|\varphi\|_{2}
$$

for all $t>0$ and $\varphi \in L_{2}(A)$, where $r=\lambda^{-1 / 2} d^{(e)}\left(A ; \Omega^{\mathrm{c}}\right)$.

Proof This follows from the identity

$$
S_{t}^{\left(h_{0}\right)} \varphi=(\pi t)^{-1 / 2} \int_{0}^{\infty} d s e^{-(4 t)^{-1} s^{2}} \cos \left(s H_{0}^{1 / 2}\right) \varphi
$$

and Proposition 3.15.

Corollary 3.20 gives good control over the semigroups $S^{(\Phi)}$ as $\Phi \rightarrow 1$ and indirectly it gives control over the distances. We first deduce that the distances $d^{(\Phi)}(A ; B)$ become independent of the choice of $\Phi$ as $\Phi \rightarrow 1$ with $A$ and $B$ fixed. 
Lemma 3.21 Let $h \in \mathcal{C}_{l}, \lambda>0$ and $\Phi, \Psi \in \mathfrak{A}$ and assume that with $h \leq\left.\lambda l\right|_{\mathfrak{A},} 0 \leq$ $\Phi, \Psi \leq 1$ and $\operatorname{supp} \Phi$, supp $\Psi$ compact. Further let $A, B \subset X$ measurable, $\Omega \subset X$ open with $\emptyset \neq \bar{A} \subset \Omega, B \subset \operatorname{supp} \Phi \cap \operatorname{supp} \Psi$ and suppose $\left.\Phi\right|_{\Omega}=\left.\Psi\right|_{\Omega}=1$. If

$$
d^{(\Phi)}(A ; B) \vee d^{(\Psi)}(A ; B) \leq \lambda^{-1 / 2} d^{(e)}\left(A ; \Omega^{\mathrm{c}}\right)
$$

then $d^{(\Phi)}(A ; B)=d^{(\Psi)}(A ; B)$.

Proof It follows from Proposition 3.15 that

$$
\cos \left(t H_{\Phi 0}^{1 / 2}\right) \varphi=\cos \left(t H_{0}^{1 / 2}\right) \varphi=\cos \left(t H_{\Psi 0}^{1 / 2}\right) \varphi
$$

for all $t \in \mathbf{R}$ with $|t| \leq \lambda^{-1 / 2} d^{(e)}\left(A ; \Omega^{\mathrm{c}}\right)$ and $\varphi \in L_{2}(A)$. Hence it follows as in the proof of Corollary 3.20 that

$$
\left\|S_{t}^{(\Phi)} \varphi-S_{t}^{(\Psi)} \varphi\right\|_{2} \leq 2 e^{-(4 t)^{-1} r^{2}}\|\varphi\|_{2}
$$

for all $t>0$ and $\varphi \in L_{2}(A)$, where $r=\lambda^{-1 / 2} d^{(e)}\left(A ; \Omega^{\mathrm{c}}\right)$. In particular,

$$
\left|\left(\mathbb{1}_{B}, S_{t}^{(\Phi)} \mathbb{1}_{A}\right)-\left(\mathbb{1}_{B}, S_{t}^{(\Psi)} \mathbb{1}_{A}\right)\right| \leq 2 e^{-(4 t)^{-1} r^{2}}|A|^{1 / 2}|B|^{1 / 2}
$$

and

$$
\begin{aligned}
\left(\mathbb{1}_{B}, S_{t}^{(\Phi)} \mathbb{1}_{A}\right) & \leq\left(\mathbb{1}_{B}, S_{t}^{(\Psi)} \mathbb{1}_{A}\right)+2 e^{-(4 t)^{-1} r^{2}}|A|^{1 / 2}|B|^{1 / 2} \\
& \leq e^{-(4 t)^{-1} d^{(\Psi)}(A ; B)^{2}}|A|^{1 / 2}|B|^{1 / 2}+2 e^{-(4 t)^{-1} r^{2}}|A|^{1 / 2}|B|^{1 / 2} \\
& \leq 3|A|^{1 / 2}|B|^{1 / 2} e^{-(4 t)^{-1} d^{(\Psi)}(A ; B)^{2}}
\end{aligned}
$$

for all $t>0$, where we used the Davies-Gaffney bounds of Theorem 2.1 in the second step. It follows by Corollary 3.14 and Lemma 3.1 that $d^{(\Phi)}(A ; B) \geq d^{(\Psi)}(A ; B)$. The converse inequality is valid by a similar argument. Hence the distances are equal.

Identification of the limit of the distances corresponding to the localizations relies on the construction of certain cut-off functions and this construction is dependent on the topological assumption. The subsequent argument follows similar reasoning in [BM95], Section 3, and [Stu95], Appendix A.

Lemma 3.22 Let $y \in X$ and define $\psi: X \rightarrow[0, \infty\rangle$ by $\psi(x)=d^{(e)}(x ; y)$. Then $\psi \wedge N \in$ $D_{0}(l)$ for all $N \in \mathbf{N}$.

Proof Since $X$ is separable there are $y_{1}, y_{2}, \ldots \in X$ such that $\left\{y_{k}: k \in \mathbf{N}\right\}$ dense is in $X$. Let $n \in \mathbf{N}$. Then $X=\bigcup_{k=1}^{\infty} B^{(e)}\left(y_{k} ; n^{-1}\right)$. Let $k \in \mathbf{N}$. By definition of $d^{(e)}$ there exists a $\psi_{n k} \in D_{0}(l) \cap C_{\mathrm{b}}(X)$ such that $\psi_{n k}(y)-\psi_{n k}\left(y_{k}\right) \geq d^{(e)}\left(y ; y_{k}\right)-n^{-1}$. Then

$$
\begin{aligned}
\psi_{n k}(x)+d^{(e)}(x ; y) & \leq \psi_{n k}(x)-\psi_{n k}\left(y_{k}\right)+\psi_{n k}\left(y_{k}\right)+d^{(e)}\left(y ; y_{k}\right)-d^{(e)}\left(y ; y_{k}\right)+d^{(e)}(x ; y) \\
& \leq d^{(e)}\left(x ; y_{k}\right)+\psi_{n k}(y)+n^{-1}+d^{(e)}\left(x ; y_{k}\right) \leq \psi_{n k}(y)+3 n^{-1}
\end{aligned}
$$

for all $x \in B\left(y_{k} ; n^{-1}\right)$. Set $\tilde{\psi}_{n k}=\left(\psi_{n k}(y)-\psi_{n k}\right) \vee 0$. Then $\tilde{\psi}_{n k} \in D_{0}(l)$. Since $\psi_{n k}(y)-$ $\psi_{n k}(x) \leq d^{(e)}(x ; y)$ for all $x \in X$ one has

$$
0 \leq \tilde{\psi}_{n k}(x) \leq d^{(e)}(x ; y)
$$

for all $x \in X$. Moreover, if $x \in B\left(y_{k} ; n^{-1}\right)$ then

$$
\tilde{\psi}_{n k}(x) \geq \psi_{n k}(y)-\psi_{n k}(x) \geq d^{(e)}(x ; y)-3 n^{-1} .
$$

Hence $\psi=\sup _{n \in \mathbf{N}} \sup _{k \in \mathbf{N}} \tilde{\psi}_{n k}$ and the lemma follows from Lemma 3.3. 
Corollary 3.23 If $K \subset X$ is compact and $\varepsilon>0$ then there exists a $\psi \in D(l) \cap C_{c}(X)$ such that $0 \leq \psi \leq 1,\left.\psi\right|_{K}=1$ and $\left\|\widehat{\mathcal{I}}_{\psi}^{(l)}\right\| \mid \leq \varepsilon$.

Proof We may assume that $K \neq \emptyset$. Let $y \in K$. There exists an $M>0$ such that $d^{(e)}(x ; y) \leq M$ for all $x \in K$. Define $\psi(x)=0 \vee\left(N-\varepsilon^{1 / 2} d^{(e)}(x ; y)\right) \wedge 1$ where $N=$ $1+\varepsilon^{1 / 2} M$. Then the corollary follows from Lemma 3.22 .

At this point we are prepared to prove the second statement in Theorem 2.2. The first step in the proof is to identify the limit of the distances corresponding to the localizations as $\Phi$ increases to the identity.

Lemma 3.24 Let $h \in \mathcal{C}_{l}$. Fix $x \in X$. Let $\Phi_{1}, \Phi_{2}, \ldots \in \mathfrak{A}$ be such that $0 \leq \Phi_{1} \leq \Phi_{2} \leq$ $\ldots \leq 1$, suppose $\left.\Phi_{n}\right|_{B^{(e)}(x ; n)}=1$ and $\operatorname{supp} \Phi_{n}$ is compact for all $n \in \mathbf{N}$. Let $A, B \subset X$ be relatively compact and measurable. Then $d^{\left(h_{0}\right)}(A ; B)=d^{\left(\Phi_{n}\right)}(A ; B)$ for all large $n \in \mathbf{N}$.

Proof One has $d^{\left(h_{0}\right)}(A ; B) \leq d^{\left(\Phi_{n+1}\right)}(A ; B) \leq d^{\left(\Phi_{n}\right)}(A ; B)$ for all $n \in \mathbf{N}$ by (6) , Corollary 3.23 and Proposition 5.1 of [ERSZ05]. Hence

$$
d^{\left(h_{0}\right)}(A ; B) \leq \inf _{n \in \mathbf{N}} d^{\left(\Phi_{n}\right)}(A ; B)=\lim _{n \rightarrow \infty} d^{\left(\Phi_{n}\right)}(A ; B)
$$

Let $M \in[0, \infty\rangle$ and suppose that $M \leq \inf _{n \in \mathbf{N}} d^{\left(\Phi_{n}\right)}(A ; B)$. Let $\varepsilon \in\langle 0,1]$. By Corollary 3.23 there exists a $\chi \in D(l) \cap C_{c}(X)$ such that $0 \leq \chi \leq 1,\left.\chi\right|_{A \cup B}=1$ and $\left\|\widehat{\mathcal{I}}_{\chi}^{(l)} \mid\right\| \leq \varepsilon^{2}$. There is an $n \in \mathbf{N}$ such that $\operatorname{supp} \chi \subset B^{(e)}(x ; n)$. Then $M \leq d^{\left(\Phi_{n}\right)}(A ; B)$, so by [ERSZ05], Lemma 4.2.III, there exists a $\psi \in D_{0}\left(h_{\Phi_{n} 0}\right)$ such that $0 \leq \psi \leq M,\left.\psi\right|_{B}=0$ and $\left.\psi\right|_{A} \geq M-\varepsilon$. Then $\chi \psi \in D\left(h_{\Phi_{n} 0}\right) \cap L_{\infty}$. Moreover, it follows from Corollary 3.19 and [ERSZ05], Lemma 3.3.II, that

$$
\begin{aligned}
\left\|\widehat{\mathcal{I}}_{\chi \psi}^{\left(h_{0}\right)}\right\|\|=\| \widehat{\mathcal{I}}_{\chi \psi}^{\left(\Phi_{n}\right)}\|\| & \leq(1+\varepsilon)\|\chi\|_{\infty}^{2}\left\|\widehat{\mathcal{I}}_{\psi}^{\left(\Phi_{n}\right)}\right\|\left\|+\left(1+\varepsilon^{-1}\right)\right\| \psi\left\|_{\infty}^{2}\right\| \widehat{\mathcal{I}}_{\chi}^{\left(\Phi_{n}\right)}\|\| \\
& \leq(1+\varepsilon)+\left(1+\varepsilon^{-1}\right) M^{2} \lambda\left\|\widehat{\mathcal{I}}_{\chi}^{(l)}\right\| \| \leq 1+N \varepsilon
\end{aligned}
$$

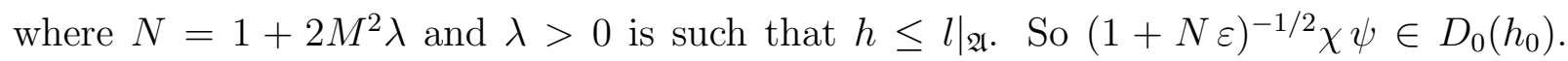
Therefore

$$
(1+N \varepsilon)^{-1 / 2}(M-\varepsilon) \leq d_{(1+N \varepsilon)^{-1 / 2} \chi \psi}(A ; B) \leq d^{\left(h_{0}\right)}(A ; B)
$$

and $M \leq d^{\left(h_{0}\right)}(A ; B)$. Thus

$$
d^{\left(h_{0}\right)}(A ; B)=\inf _{n \in \mathbf{N}} d^{\left(\Phi_{n}\right)}(A ; B)=\lim _{n \rightarrow \infty} d^{\left(\Phi_{n}\right)}(A ; B)
$$

If $d^{\left(h_{0}\right)}(A ; B)=\infty$ then obviously $d^{\left(h_{0}\right)}(A ; B)=d^{\left(\Phi_{n}\right)}(A ; B)$ for all $n \in \mathbf{N}$. Alternatively, if $d^{\left(h_{0}\right)}(A ; B)<\infty$ then the lemma follows from Lemma 3.21 together with (12).

At this point we have control over the limits of the semigroups $S^{(\Phi)}$ and the distances $d^{(\Phi)}$ associated with the truncated forms.

Proof of Theorem 2.2 Since, by hypothesis, $h \in \mathcal{C}_{l}$ and $l$ satisfies Condition L the foregoing results are applicable. Let $\lambda>0$ be such that $h \leq\left.\lambda l\right|_{\mathfrak{A} \text {. }}$

It follows from the Davies-Gaffney bounds Theorem 2.1 that

$$
t \log \left(\mathbb{1}_{A}, S_{t}^{\left(h_{0}\right)} \mathbb{1}_{B}\right) \leq-4^{-1} d^{\left(h_{0}\right)}(A ; B)^{2}
$$


for all $t>0$. If $d^{\left(h_{0}\right)}(A ; B)=\infty$ then (7) is obviously valid. So we may assume that $d^{\left(h_{0}\right)}(A ; B)<\infty$. Then in particular $A \neq \emptyset$.

Fix $x \in X$. Let $\Phi_{1}, \Phi_{2}, \ldots \in \mathfrak{A}$ be such that $0 \leq \Phi_{1} \leq \Phi_{2} \leq \ldots \leq 1,\left.\Phi_{n}\right|_{B^{(e)}(x ; n)}=1$ and $\operatorname{supp} \Phi_{n}$ is compact for all $n \in \mathbf{N}$. Let $\varepsilon>0$. By Lemma 3.24 there exists an $n \in \mathbf{N}$ such that $d^{\left(h_{0}\right)}(A ; B)=d^{\left(\Phi_{n}\right)}(A ; B)$ and $r^{2}=\lambda^{-1} d^{(e)}\left(A ; B^{(e)}(x ; n)^{\mathrm{c}}\right)^{2}>d^{\left(h_{0}\right)}(A ; B)^{2}+8 \varepsilon$. Then

$$
\left|\left(\mathbb{1}_{A}, S_{t}^{\left(h_{0}\right)} \mathbb{1}_{B}\right)-\left(\mathbb{1}_{A}, S_{t}^{\left(\Phi_{n}\right)} \mathbb{1}_{B}\right)\right| \leq 2 e^{-(4 t)^{-1} r^{2}}|A|^{1 / 2}|B|^{1 / 2}
$$

for all $t>0$ by Corollary 3.20. Moreover, by Corollary 3.14 there exists a $t_{0}>0$ such that

$$
t \log \left(\mathbb{1}_{A}, S_{t}^{\left(\Phi_{n}\right)} \mathbb{1}_{B}\right) \geq-4^{-1} d^{\left(\Phi_{n}\right)}(A ; B)^{2}-\varepsilon
$$

for all $t \in\left\langle 0, t_{0}\right]$. Then

$$
\begin{aligned}
\left(\mathbb{1}_{A}, S_{t}^{\left(h_{0}\right)} \mathbb{1}_{B}\right) & \geq\left(\mathbb{1}_{A}, S_{t}^{\left(\Phi_{n}\right)} \mathbb{1}_{B}\right)-2 e^{-(4 t)^{-1} r^{2}}|A|^{1 / 2}|B|^{1 / 2} \\
& \geq e^{-(4 t)^{-1} d^{\left(\Phi_{n}\right)}(A ; B)^{2}} e^{-\varepsilon t^{-1}}-2 e^{-(4 t)^{-1} r^{2}}|A|^{1 / 2}|B|^{1 / 2} \\
& \geq e^{-(4 t)^{-1} d^{\left(h_{0}\right)}(A ; B)^{2}} e^{-\varepsilon t^{-1}}\left(1-2 e^{-\varepsilon t^{-1}}|A|^{1 / 2}|B|^{1 / 2}\right)
\end{aligned}
$$

for all $t \in\left\langle 0, t_{0}\right]$, where we used $d^{\left(h_{0}\right)}(A ; B)^{2}+4 \varepsilon-r^{2} \leq-4 \varepsilon$ in the last step. There is a $t_{1}>0$ such that $2 e^{-\varepsilon t_{1}^{-1}}|A|^{1 / 2}|B|^{1 / 2} \leq 4^{-1}$. Then

$$
\left(\mathbb{1}_{A}, S_{t}^{\left(h_{0}\right)} \mathbb{1}_{B}\right) \geq 2^{-1} e^{-(4 t)^{-1} d^{\left(h_{0}\right)}(A ; B)^{2}} e^{-\varepsilon t^{-1}}
$$

and

$$
t \log \left(\mathbb{1}_{A}, S_{t}^{\left(h_{0}\right)} \mathbb{1}_{B}\right) \geq-4^{-1} d^{\left(h_{0}\right)}(A ; B)^{2}-\varepsilon-t \log 2
$$

for all $t \in\left\langle 0, t_{0} \wedge t_{1}\right]$. This completes the proof of Theorem 2.2 .

Proof of Corollary 2.4 We may assume that $|A| \neq 0$ and $|B| \neq 0$. If $\varphi, \psi \in L_{2}(X)$ then $P_{A} \psi \in L_{2}(A)$ and $P_{B} \varphi \in L_{2}(B)$. So by the Davies-Gaffney bounds of Theorem 2.1] one deduces that

$$
\begin{aligned}
\left|\left(\psi, P_{A} S_{t} P_{B} \varphi\right)\right| & =\left|\left(P_{A} \psi, S_{t} P_{B} \varphi\right)\right| \\
& \leq e^{-(4 t)^{-1} d(A ; B)^{2}}\left\|P_{A} \psi\right\|_{2}\left\|P_{B} \varphi\right\|_{2} \leq e^{-(4 t)^{-1} d(A ; B)^{2}}\|\psi\|_{2}\|\varphi\|_{2} .
\end{aligned}
$$

Hence

$$
\limsup _{t \downarrow 0} t \log \left\|P_{A} S_{t} P_{B}\right\|_{2 \rightarrow 2} \leq-4^{-1} d(A ; B)^{2}
$$

So it remains to show that

$$
-4^{-1} d(A ; B)^{2} \leq \liminf _{t \downarrow 0} t \log \left\|P_{A} S_{t} P_{B}\right\|_{2 \rightarrow 2}
$$

Let $A_{0} \subseteq A$ and $B_{0} \subseteq B$ be measurable and relatively compact and assume that $\left|A_{0}\right|>0$ and $\left|B_{0}\right|>0$. Then

$$
\left(\mathbb{1}_{A_{0}}, S_{t} \mathbb{1}_{B_{0}}\right)=\left(\mathbb{1}_{A_{0}}, P_{A} S_{t} P_{B} \mathbb{1}_{B_{0}}\right) \leq\left|A_{0}\right|^{1 / 2}\left\|P_{A} S_{t} P_{B}\right\|_{2 \rightarrow 2}\left|B_{0}\right|^{1 / 2}
$$


for all $t>0$. So

$$
\begin{aligned}
\liminf _{t \downarrow 0} t \log \left\|P_{A} S_{t} P_{B}\right\|_{2 \rightarrow 2} & \geq \liminf _{t \downarrow 0}\left(t \log \left(\mathbb{1}_{A_{0}}, S_{t} \mathbb{1}_{B_{0}}\right)-2^{-1} t \log \left(\left|A_{0}\right|\left|B_{0}\right|\right)\right) \\
& =-4^{-1} d\left(A_{0} ; B_{0}\right)^{2}
\end{aligned}
$$

by Theorem 2.2. Now fix $x \in X$ and choose $A_{0}=A \cap B^{(e)}(x ; n)$ and $B_{0}=B \cap B^{(e)}(x ; n)$ with $n \in \mathbf{N}$ large enough. Then

$$
\begin{aligned}
\liminf _{t \downarrow 0} t \log \left\|P_{A} S_{t} P_{B}\right\|_{2 \rightarrow 2} & \geq-\lim _{n \rightarrow \infty} 4^{-1} d\left(A \cap B^{(e)}(x ; n) ; B \cap B^{(e)}(x ; n)\right)^{2} \\
& =-4^{-1} d(A ; B)^{2}
\end{aligned}
$$

where the equality follows from Lemma 3.4. This proves (13) and the corollary.

Now we turn to the proof of Theorem 2.5. First we use the continuity assumption 2.5] of the theorem to identify the distance.

Lemma 3.25 If $D_{0}(l) \subset C(X)$ then $d^{(l)}(A ; B)=d^{(e)}(A ; B)$ for all non-empty open subsets $A, B \subset X$.

Proof Let $M \in\left[0, d^{(l)}(A ; B)\right] \cap \mathbf{R}$ and $\varepsilon>0$. Then by [ERSZ05], Lemma 4.2.III, there exists a $\psi \in D_{0}(l)$ such that $\left.\psi\right|_{B}=0$ almost everywhere and $\left.\psi\right|_{A} \geq M-\varepsilon$ almost everywhere. Since $D_{0}(l) \subset C(X)$ it follows that $\psi \in D(l)_{\text {loc }} \cap C_{\mathrm{b}}(X)$. Moreover, since $A$ and $B$ are open one has $\left.\psi\right|_{B}=0$ and $\left.\psi\right|_{A} \geq M-\varepsilon$ pointwise. So $|\psi(x)-\psi(y)| \geq M-\varepsilon$ for all $x \in A$ and $y \in B$. It follows that $d^{(e)}(A ; B) \geq M-\varepsilon$ and $d^{(e)}(A ; B) \geq d^{(l)}(A ; B)$. The converse inequality is trivial (see Lemma 3.11II).

Proof of Theorem 2.5 Let $x_{0}, y_{0} \in X$ and $\varepsilon \in\langle 0,1]$. Choose $A=B^{(e)}\left(x_{0} ; \varepsilon\right)$ and $B=B^{(e)}\left(y_{0} ; \varepsilon\right)$. Let $t \in\left\langle 0,4^{-1} T\right]$. Then by assumption $\amalg$ of Theorem 2.5 and symmetry of the kernel

$$
\begin{aligned}
K_{t}\left(x_{0} ; y_{0}\right) & \leq K_{(1+\varepsilon) t}\left(x_{0} ; y\right)(1+\varepsilon)^{\nu} e^{\omega\left(1+\varepsilon t^{-1}\right)} \\
& \leq K_{(1+\varepsilon)^{2} t}(x ; y)(1+\varepsilon)^{2 \nu} e^{2 \omega\left(1+\varepsilon t^{-1}\right)}
\end{aligned}
$$

for all $x \in A$ and $y \in B$. So

$$
\begin{aligned}
K_{t}\left(x_{0} ; y_{0}\right) & \leq|A|^{-1}|B|^{-1} \int_{A} d x \int_{B} d y K_{(1+\varepsilon)^{2} t}(x ; y)(1+\varepsilon)^{2 \nu} e^{2 \omega\left(1+\varepsilon t^{-1}\right)} \\
& =|A|^{-1}|B|^{-1}(1+\varepsilon)^{2 \nu} e^{2 \omega\left(1+\varepsilon t^{-1}\right)}\left(\mathbb{1}_{A} S_{(1+\varepsilon)^{2} t^{(l)}} \mathbb{1}_{B}\right)
\end{aligned}
$$

Therefore

$$
t \log K_{t}\left(x_{0} ; y_{0}\right) \leq t \log \left(\mathbb{1}_{A} S_{(1+\varepsilon)^{2} t^{2}}^{(l)} \mathbb{1}_{B}\right)-t \log (|A||B|)+2 \nu t \log (1+\varepsilon)+2 \omega t+2 \omega \varepsilon
$$

for all $t \in\left\langle 0,4^{-1} T\right]$. Since $|A|,|B|>0$ one deduces from Corollary 2.3 that

$\limsup _{t \downarrow 0} t \log K_{t}\left(x_{0} ; y_{0}\right) \leq-4^{-1}(1+\varepsilon)^{-2} d^{(l)}(A ; B)+2 \omega \varepsilon=-4^{-1}(1+\varepsilon)^{-2} d^{(e)}(A ; B)+2 \omega \varepsilon$ 
where we used Lemma 3.25 in the last step. But

$$
d^{(e)}\left(x_{0}, y_{0}\right)-2 \varepsilon \leq d^{(e)}\left(B^{(e)}\left(x_{0} ; \varepsilon\right) ; B^{(e)}\left(y_{0} ; \varepsilon\right)\right) \leq d^{(e)}\left(x_{0}, y_{0}\right)+2 \varepsilon
$$

by the triangle inequality. So taking the limit $\varepsilon \downarrow 0$ one establishes that

$$
\limsup _{t \downarrow 0} t \log K_{t}\left(x_{0} ; y_{0}\right) \leq-4^{-1} d^{(e)}\left(x_{0}, y_{0}\right)
$$

Since, by an analogous argument,

$$
K_{t}\left(x_{0} ; y_{0}\right) \geq K_{(1-\varepsilon)^{2} t}(x ; y)(1+\varepsilon)^{-2 \nu} e^{-3 \omega\left(1+\varepsilon t^{-1}\right)}
$$

for all $t \in\langle 0, T], \varepsilon \in\langle 0,1 / 2\rangle, x \in B^{(e)}\left(x_{0} ; \varepsilon\right)$ and $y \in B^{(e)}\left(y_{0} ; \varepsilon\right)$ one deduces similarly that

$$
\liminf _{t \downarrow 0} t \log K_{t}\left(x_{0} ; y_{0}\right) \geq-4^{-1} d^{(e)}\left(x_{0}, y_{0}\right)
$$

So $\lim _{t \downarrow 0} t \log K_{t}\left(x_{0} ; y_{0}\right)=-4^{-1} d^{(e)}\left(x_{0}, y_{0}\right)$ and Theorem 2.5 follows.

\section{Applications}

Theorems 2.2 and 2.5 have a broad range of applications to second-order, divergence-form, elliptic operators both degenerate and non-degenerate. First we discuss the application to operators on $\mathbf{R}^{d}$.

Let $c_{i j}$ be bounded real-valued measurable functions on $\mathbf{R}^{d}$ and assume that the $d \times d$ matrix $C=\left(c_{i j}\right)$ is symmetric and positive-definite almost-everywhere. Define $h$ by

$$
h(\varphi)=\sum_{i, j=1}^{d}\left(\partial_{i} \varphi, c_{i j} \partial_{j} \varphi\right)
$$

where $\partial_{i}=\partial / \partial x_{i}$ and $D(h)=W^{1,2}\left(\mathbf{R}^{d}\right)$. Then $h$ is a positive form which is strongly local and Markovian. It is not in general closed or even closable. A characterization of closable forms in one dimension can be found in [FOT94, Theorem 3.1.6, and sufficient conditions in higher dimensions are given in [FOT94], Section 3.1 (see also [RW85] or [MR92, Chapter II). Nevertheless, $h \leq \lambda l$ where $\lambda$ denotes the essential supremum of the matrix norms $\|C(x)\|$ and $l$ is the form of the usual Laplacian on $\mathbf{R}^{d}$, i.e., $D(l)=W^{1,2}\left(\mathbf{R}^{d}\right)$ and

$$
l(\varphi)=\sum_{i=1}^{d}\left\|\partial_{i} \varphi\right\|_{2}^{2}=\|\nabla \varphi\|_{2}^{2}
$$

for all $\varphi \in D(l)$.

First, consider the case of strongly elliptic operators, i.e., assume that $C \geq \mu I$ almost everywhere, with $\mu>0$. Then one has $\lambda l \geq h \geq \mu l$ and the form $h$ is closed on $W^{1,2}\left(\mathbf{R}^{d}\right)$. It follows readily that $h$ is a diffusion satisfying Condition $L$ and the distance $d^{(e)}$ is the Riemannian distance corresponding to the metric $C^{-1}$. Moreover, Conditions 【 and 【 of Theorem 2.5 are valid. The latter condition follows from the parabolic Harnack inequality (see, for example, [SC02], Chapter 5 and in particular Corollary 5.4.6). Therefore Varadhan's small time asymptotic result (11) follows for each strongly elliptic operator by Corollary 2.3 and Theorem 2.5. 
Secondly, consider degenerate operators. Thus $C \geq 0$ almost everywhere but this is the only coercivity condition. Nevertheless, $h$ is a strongly local Markovian form and $h \leq\left.\lambda l\right|_{\mathfrak{A}}$. Therefore Theorem 2.2 is directly applicable to $h$ with no further assumptions on the coefficients. It then follows that $h_{0}$ is strongly local and the asymptotic identification (17) is valid. Further detail on the asymptotic behaviour requires more detailed analysis of the set-theoretic distance $d^{\left(h_{0}\right)}(A ; B)$. In the degenerate situation the set $U_{h}=\left\{x \in \mathbf{R}^{d}\right.$ : $\|C(x)\|=0\}$ may have non-zero measure and the diffusion associated with the relaxation $h_{0}$ occurs on the closure of the complement $\mathbf{R}^{d} \backslash U_{h}$. In fact the effective space of the diffusion can be smaller since sets of capacity zero act as obstacles [RS05].

The properties of the distance $d^{(h)}(A ; B)$ can be understood for non-degenerate, or weakly degenerate, elliptic operators. In particular it can be analyzed for subelliptic operators in quite general situations.

Let $M$ be a Riemannian manifold and $X_{1}, \ldots, X_{n}$ smooth vector fields on $M$. Each such vector field $X$ defines a closed linear partial differential operator, also denoted by $X$, on $L_{2}(M)$. Now consider the form

$$
h(\varphi)=\sum_{i=1}^{d}\left\|X_{i} \varphi\right\|_{2}^{2}=\||\nabla \varphi|\|_{2}^{2}
$$

with $D(h)=\bigcap_{i=1}^{n} D\left(X_{i}\right)$ and $\nabla \varphi=\left(X_{1} \varphi, \ldots, X_{n} \varphi\right)$. The form is automatically closed regular and strongly local. Next assume the form is densely-defined and that the vector fields satisfy the Hörmander condition of order $r$. It follows readily that $\psi \in D_{0}(h)$ if and only if $|\nabla \psi| \in L_{\infty}(M)$ and $|\nabla \psi|^{2} \leq 1$ almost everywhere. But it then follows from [RS76, Theorem 17, that $\psi$ is locally Lipschitz. In fact it has a Lipschitz derivative of order $1 / r$. Consequently, $D_{0}(l) \subseteq C(M)$. Thus the first assumption of Theorem 2.5 is verified in quite general circumstances. The second assumption is also verifiable in many situations as it is a consequence of a parabolic Harnack inequality. As a specific illustration we give an application to subelliptic operators on Lie groups.

Proposition 4.1 Let $X_{1}, \ldots, X_{n}$ be right invariant vector fields on a Lie group $G$ which satisfy the Hörmander condition, i.e., the vector fields generate the Lie algebra of $G$. For all $i, j \in\{1, \ldots, n\}$ let $c_{i j}=c_{j i} \in L_{\infty}(G)$ be real valued and assume there is a $\mu>0$ such that $\left(c_{i j}(g)\right) \geq \mu I$ for almost every $g \in G$. Define the quadratic form $h$ on $L_{2}(G)$ by

$$
h(\varphi)=\sum_{i, j=1}^{n}\left(X_{i} \varphi, c_{i j} X_{j} \varphi\right)
$$

with form domain $D(h)=\bigcap_{i=1}^{n} D\left(X_{i}\right)$. Then the form $h$ is a diffusion satisfying Condition $\mathrm{L}$ and the assumptions of Theorem 2.5 are satisfied. Hence

$$
\lim _{t \downarrow 0} t \log K_{t}\left(g_{1} ; g_{2}\right)=-4^{-1} d\left(g_{1} ; g_{2}\right)^{2}
$$

for all $g_{1}, g_{2} \in G$, where $K$ is the kernel of the semigroup generated by the operator $H$ associated to the form $h$ and

$$
d\left(g_{1} ; g_{2}\right)=\sup \left\{\left|\psi\left(g_{1}\right)-\psi\left(g_{2}\right)\right|: \psi \in D(h)_{\mathrm{loc}} \cap C_{\mathrm{b}}(G) \text { and }\left\|\left|\widehat{\mathcal{I}}_{\psi}\right|\right\| \leq 1\right\} .
$$


Proof It is straightforward to check that $h$ is a diffusion. Note also that $h$ is bounded above and below by multiples of the form

$$
l(\varphi)=\sum_{i=1}^{n}\left\|X_{i} \varphi\right\|_{2}^{2}
$$

associated with the sublaplacian given by the vector fields. Therefore the distances $d^{(e)}$ for $l$ and $d$ (defined by (14) ) for $h$ are equivalent. Condition $\mathrm{L}$ and the first assumption of Theorem 2.5 for the form $l$ and then also for the form $h$ follow since the vector fields satisfy the Hörmander condition. The second assumption of the theorem follows from the parabolic Harnack inequality (again see [SC02], Chapter 5 and in particular Corollary 5.4.6, or the first remark following Theorem 4.4 on page 35-36 in [SC92]).

Next we note that Norris' result on the small time behaviour of the Laplace-Beltrami operator can also be deduced from our results. Let $M$ be a $d$-dimensional Lipschitz Riemannian manifold with Borel measure $\mu$ which under some chart is locally equivalent with the Lebesgue measure. Further let $l$ denote the Dirichlet form

$$
l(\varphi)=\int_{M} d \mu|\nabla \varphi|^{2}
$$

where $\nabla$ denotes the usual gradient and $D(l)=W^{1,2}(M)$. The corresponding self-adjoint operator on $L_{2}(M)$ is the Laplace-Beltrami operator for $M$. Using a local coordinate chart one easily argues that $\psi \in W_{\text {loc }}^{1,2} \cap L_{\infty}$ and $\left\|\mid \widehat{\mathcal{I}}_{\psi}^{(l)}\right\| \| \leq 1$ if and only if $\psi \in W^{1, \infty}$ and $\|\nabla \psi\|_{\infty} \leq 1$. In particular $D_{0}(l) \subseteq C(M)$. Since the Riemannian distance on $M$ is given by

$$
d(x ; y)=\sup \left\{|\psi(x)-\psi(y)|: \psi \in W^{1, \infty},\|\nabla \psi\|_{\infty} \leq 1\right\}
$$

it follows that Condition $\mathrm{L}$ is satisfied. Moreover, the parabolic Harnack inequality is valid (and is used in the proof of Norris (see [Nor97, page 87)). Therefore, by the above reasoning, one can apply Theorem 2.5 to establish

$$
\lim _{t \downarrow 0} t \log K_{t}(x ; y)=-4^{-1} d(x ; y)^{2}
$$

for all $x, y \in M$. Note that as in Norris' argument this proof requires neither smoothness nor completeness of $M$.

Finally we note that the asymptotic estimates can be extended to operators with lower order terms by various arguments such as perturbation theory. For example, if $h$ is a Dirichlet form and $v$ the form of a real, positive, bounded multiplication operator $V$ then the Trotter product formula

$$
S_{t}^{(h+v)}=\lim _{n \rightarrow \infty}\left(S_{t / n}^{(h)} S_{t / n}^{(v)}\right)^{n}
$$

Therefore one deduces from positivity of the semigroups that

$$
S_{t}^{(h)} e^{-t\|V\|_{\infty}} \leq S_{t}^{(h+v)} \leq S_{t}^{(h)}
$$

for all $t>0$. Hence

$$
\lim _{t \downarrow 0} t \log \left(\mathbb{1}_{A}, S_{t}^{(h+v)} \mathbb{1}_{B}\right)=\lim _{t \downarrow 0} t \log \left(\mathbb{1}_{A}, S_{t}^{(h)} \mathbb{1}_{B}\right)
$$


for all relatively compact measurable subsets $A$ and $B$. Thus the small time asymptotics is independent of $v$.

Next, if $h$ is a regular Dirichlet for and $v$ is the form of the operator of multiplication by a real, positive, locally bounded measurable function $V$, then obviously $h+v$ is densily defined. But in addition $h+v$ is closed by Sim78. Theorem 4.1, since the closed forms $h+v_{n}$ converge monotonically upwards to $h+v$, where $v_{n}$ denotes the form of the operator of multiplication by the bounded function $V \mathbb{1}_{X_{n}}$ and $X_{1} \subset X_{2} \subset \ldots$ are measurable subsets of $X$ with $X=\bigcup_{n=1}^{\infty} X_{n}$.

These observations extend to the following result.

Proposition 4.2 Let $h$ be a positive form satisfying the hypotheses of Theorem [2.2 and with $D(h)=D(l)$. Further, let $v$ be the form of the operator of multiplication by a real, positive, locally bounded measurable function $V$. Then

$$
(h+v)_{0}=h_{0}+v=\mathrm{r}_{\varepsilon \rightarrow 0} \lim _{\varepsilon}\left(h_{\varepsilon}+v\right)
$$

and

$$
\lim _{t \downarrow 0} t \log \left(\mathbb{1}_{A}, S_{t}^{\left((h+v)_{0}\right)} \mathbb{1}_{B}\right)=-4^{-1} d^{\left(h_{0}\right)}(A ; B)^{2}
$$

for all relatively compact measurable subsets $A$ and $B$.

Proof First, $h_{0}+v$ is closed, densely-defined and $h_{0}+v \leq h+v \leq h_{\varepsilon}+v$ for all $\varepsilon>0$, where in the second inequality we use the additional assumption $D(h)=D(l)$. (Recall that by definition $D\left(h_{\varepsilon}\right)=D(l)$.) Therefore $h_{0}+v \leq(h+v)_{0} \leq \hat{h}$ where $\hat{h}=$ r. $\lim _{\varepsilon \downarrow 0}\left(h_{\varepsilon}+v\right)$. We now establish the first statement of the proposition by proving that $\hat{h}=h_{0}+v$.

Let $\Omega_{1} \subset \Omega_{2} \subset \ldots$ be open relatively compact subsets of $X$ such that $X=\bigcup_{n=1}^{\infty} \Omega_{n}$. For all $n \in \mathbf{N}$ let $v_{n}$ denote the form of the operator of multiplication by the bounded function $V \mathbb{1}_{\Omega_{n}}$. Let $\varepsilon>0$. Set $h_{\varepsilon, n}=h_{\varepsilon}+v_{n}$ for all $n \in \mathbf{N}$. Then $S_{t}^{\left(h_{\varepsilon, n}\right)} \leq S_{t}^{\left(h_{\varepsilon}\right)}$ for all $t>0$ by (15) and

$$
\left(\mathbb{1}_{A}, S_{t}^{\left(h_{\varepsilon, n}\right)} \mathbb{1}_{B}\right) \leq\left(\mathbb{1}_{A}, S_{t}^{\left(h_{\varepsilon}\right)} \mathbb{1}_{B}\right) \leq e^{-(\lambda+\varepsilon)^{-1 / 2} d^{(e)}(A ; B)^{2}(4 t)^{-1}}|A|^{1 / 2}|B|^{1 / 2}
$$

for all non-empty open $A, B \subset X$ and $t>0$ where the second bound follows by Lem-

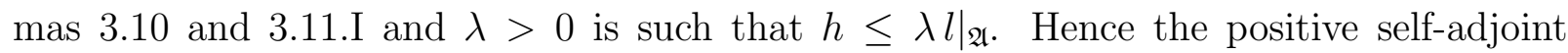
operator $H_{\varepsilon, n}$ associated with $h_{\varepsilon, n}$ has a finite speed of propagation. Explicitly

$$
\left(\psi, \cos \left(t H_{\varepsilon, n}^{1 / 2}\right) \varphi\right)=0
$$

for all non-empty open $A, B \subset X$, all $\varphi \in L_{2}(A), \psi \in L_{2}(B)$ and all $t \in \mathbf{R}$ with $|t| \leq$ $(\lambda+\varepsilon)^{-1 / 2} d^{(e)}(A ; B)$ by ERSZ04, Lemma 3.3, and the assumptions on $h$. Then arguing as in the proof of Proposition 3.15 one deduces that

$$
\cos \left(t H_{\varepsilon, n}^{1 / 2}\right) \varphi=\cos \left(t H_{\varepsilon, m}^{1 / 2}\right) \varphi
$$

for all $n, m \in \mathbf{N}$, measurable $A \subset X, \varphi \in L_{2}(A)$ and $t \in \mathbf{R}$ with $m \geq n, \emptyset \neq \bar{A} \subset \Omega_{n}$ and $|t| \leq(\lambda+\varepsilon)^{-1 / 2} d^{(e)}\left(A ; \Omega_{n}^{\mathrm{c}}\right)$. The proof is a repetition of the arguments used to prove Proposition 3.15 but now one also uses locality of $v_{n}$ and $v_{m}$. Therefore using the representation (11) one obtains an estimate

$$
\left\|S_{t}^{\left(h_{\varepsilon, n}\right)} \varphi-S_{t}^{\left(h_{\varepsilon, m}\right)} \varphi\right\|_{2} \leq 2 e^{-(4 t)^{-1} r_{n}^{2}}\|\varphi\|_{2}
$$


for all $n, m \in \mathbf{N}$, measurable $A \subset X, \varphi \in L_{2}(A)$ and $t>0$ with $m \geq n, \emptyset \neq \bar{A} \subset \Omega_{n}$, where $r_{n}=(\lambda+\varepsilon)^{-1 / 2} d^{(e)}\left(A ; \Omega_{n}^{\mathrm{c}}\right)$. Now if $m \rightarrow \infty$ then $h_{\varepsilon, m}$ converges monotonically upward to $h_{\varepsilon}+v$. Hence $S_{t}^{\left(h_{\varepsilon, m}\right)}$ converges strongly to $S_{t}^{\left(h_{\varepsilon}+v\right)}$ by Sim78, Theorem 3.1. But $S_{t}^{\left(h_{\varepsilon}+v\right)}$ converges strongly to $S_{t}^{(\hat{h})}$ as $\varepsilon \downarrow 0$ and $S_{t}^{\left(h_{\varepsilon, n}\right)}$ converges strongly to $S_{t}^{\left(h_{0}+v_{n}\right)}$ as $\varepsilon \downarrow 0$ because $r \cdot \lim _{\varepsilon \downarrow 0} h_{\varepsilon, n}=h_{0}+v_{n}$. Combining these observations one deduces that

$$
\left\|S_{t}^{\left(h_{0}+v_{n}\right)} \varphi-S_{t}^{(\hat{h})} \varphi\right\|_{2} \leq 2 e^{-(4 t)^{-1} r_{n}^{2}}\|\varphi\|_{2}
$$

for all $n \in \mathbf{N}$, measurable $A \subset X, \varphi \in L_{2}(A)$ and $t>0$ with $\emptyset \neq \bar{A} \subset \Omega_{n}$. Finally r. $\lim _{n \rightarrow \infty}\left(h_{0}+v_{n}\right)=h_{0}+v$ by monotone convergence and as $r_{n} \rightarrow \infty$ as $n \rightarrow \infty$ one concludes that $S_{t}^{\left(h_{0}+v\right)} \varphi=S_{t}^{(\hat{h})} \varphi$ for all $\varphi \in L_{2, c}(X)$ and $t>0$. Thus $S_{t}^{\left(h_{0}+v\right)}=S_{t}^{(\hat{h})}$ for all $t>0$. Hence $\hat{h}=h_{0}+v$.

Finally we deduce from (15) that

$$
\left(\mathbb{1}_{A}, S_{t}^{(\hat{h})} \mathbb{1}_{B}\right)=\left(\mathbb{1}_{A}, S_{t}^{\left(h_{0}+v\right)} \mathbb{1}_{B}\right) \leq\left(\mathbb{1}_{A}, S_{t}^{\left(h_{0}\right)} \mathbb{1}_{B}\right) \leq e^{-d^{\left(h_{0}\right)}(A ; B)^{2}(4 t)^{-1}}|A|^{1 / 2}|B|^{1 / 2}
$$

for all non-empty open $A, B \subset X$ and $t>0$, where the last bound uses Theorem [2.1. Finally the proof of the proposition is completed by repeating the arguments used above in the proof of Theorem 2.2 with $S^{\left(h_{0}\right)}$ replaced by $S^{(\hat{h})}$ and $S^{(\Phi)}$ replaced by $S^{\left(h_{0}+v_{n}\right)}$. The equality (16) and inequality (17) replace Corollaries 3.14 and 3.20.

Note that the identification $(h+v)_{0}=h_{0}+v$ shows that $(h+v)_{0}$ is local in the sense of [FOT94].

\section{Acknowledgements}

This work was supported by the Australian Research Council (ARC) Discovery Grant DP 0451016. The greater part of this work was carried out during a visit of the first named author to the Australian National University. The work was completed whilst the first and second named authors were visiting the Centre International de Rencontres Mathématiques at the Université de la Méditerranée at Luminy.

\section{References}

[AH05] Ariyoshi, T., and Hino, M., Small-time asymptotic estimates in local Dirichlet spaces. Elec. J. Prob. 10 (2005), 1236-1259.

[Aze81] Azencott, R., Géodésiques et diffusions en temps petit, vol. 84 of Astérisque. Société Mathématique de France, Paris, 1981. Séminaire de probabilités, Université de Pais VII.

[BD58] Beurling, A., and Deny, J., Espaces de Dirichlet. I. Le cas élémentaire. Acta Math. 99 (1958), 203-224.

[BH91] Bouleau, N., and Hirsch, F., Dirichlet forms and analysis on Wiener space, vol. 14 of de Gruyter Studies in Mathematics. Walter de Gruyter \& Co., Berlin, 1991. 
[BKRR71] Bonami, A., Karoui, N., Roynette, B., and Reinhard, H., Processus de diffusion associé à un opérateur elliptique dégénéré. Ann. Inst. H. Poincaré Sect. $B$ (N.S.) 7 (1971), 31-80.

[BM95] Biroli, M., and Mosco, U., A Saint-Venant type principle for Dirichlet forms on discontinuous media. Ann. Mat. Pura Appl. 169 (1995).

[ERSZ04] Elst, A. F. M. ter, Robinson, D. W., Sikora, A., and Zhu, Y., Secondorder operators with degenerate coefficients. Research Report CASA 04-32, Eindhoven University of Technology, Eindhoven, The Netherlands, 2004.

[ERSZ05] — Dirichlet forms and degenerate elliptic operators. In KoELINK, E., Neerven, J. van, Pagter, B. De, and Sweers, G., eds., Partial Differential Equations and Functional Analysis. Birkhäuser, 2005. Philippe Clement Festschrift, to appear.

[FOT94] Fukushima, M., Oshima, Y., and Takeda, M., Dirichlet forms and symmetric Markov processes, vol. 19 of de Gruyter Studies in Mathematics. Walter de Gruyter \& Co., Berlin, 1994.

[Hin02] Hino, M., On short time asymptotic behavior of some symmetric diffusions on general state spaces. Potential Anal. 16 (2002), 249-264.

[HR03] Hino, M., and Ramírez, J. A., Small-time Gaussian behavior of symmetric diffusion semigroups. Ann. Prob. 31 (2003), 254-1295.

[Kat80] Kato, T., Perturbation theory for linear operators. Second edition, Grundlehren der mathematischen Wissenschaften 132. Springer-Verlag, Berlin etc., 1980.

[KS88] Kusuoka, S., and Stroock, D., Long time estimates for the heat kernel associated with a uniformly subelliptic symmetric second order operator. Ann. Math. 127 (1988), 165-189.

[Léa87a] LÉAnDRE, R., Majoration en temps petit de la densité d'une diffusion dégénérée. Probab. Theory Related Fields 74 (1987), 289-294.

[Léa87b] — Minoration en temps petit de la densité d'une diffusion dégénérée. J. Funct. Anal. 74 (1987), 399-414.

[LeJ78] LeJan, Y., Mesures associées à une forme de Dirichlet. Applications. Bull. Soc. Math. France 106 (1978), 61-112.

[Mol75] MolČAnov, S. A., Diffusion processes, and Riemannian geometry. Uspehi Mat. Nauk 30, No. 1(181) (1975), 3-59.

[Mos94] Mosco, U., Composite media and asymptotic Dirichlet forms. J. Funct. Anal. 123 (1994), 368-421.

[MR92] MA, Z. M., and RöcKneR, M., Introduction to the theory of (non symmetric) Dirichlet Forms. Universitext. Springer-Verlag, Berlin etc., 1992. 
[Nor97] NorRIs, J. R., Heat kernel asymptotics and the distance function in Lipschitz Riemannian manifolds. Acta Math. 179 (1997), 79-103.

[Ram01] Ramírez, J. A., Short-time asymptotics in Dirichlet spaces. Comm. Pure Appl. Math. 54 (2001), 259-293.

[Rot76] Roth, J.-P., Formule de représentation et troncature des formes de Dirichlet sur $\mathbf{R}^{m}$. In Séminaire de Théorie du Potentiel de Paris, No. 2, Lect. Notes in Math. 563, 260-274. Springer Verlag, Berlin, 1976.

[RS72] ReEd, M., and Simon, B., Methods of modern mathematical physics I. Functional analysis. Academic Press, New York etc., 1972.

[RS76] Rothschild, L. P., and Stein, E. M., Hypoelliptic differential operators and nilpotent groups. Acta Math. 137 (1976), 247-320.

[RS05] Robinson, D. W., and Sikora, A. Degenerate elliptic operators: capacity, flux and separation, 2005. Submitted to Potential Analysis.

[RW85] Röckner, M., and WiElens, N., Dirichlet forms - closability and change of speed measure. In Infinite-dimensional analysis and stochastic processes (Bielefeld, 1983), vol. 124 of Res. Notes in Math., 119-144. Pitman, Boston, 1985.

[SC92] Saloff-Coste, L., A note on Poincaré, Sobolev, and Harnack inequalities. Internat. Math. Res. Notices 1992, No. 2 (1992), 27-38.

[SC02] — Aspects of Sobolev-type inequalities. London Math. Soc. Lect. Note Series 289. Cambridge University Press, Cambridge, 2002.

[Sim77] Simon, B., Lower semicontinuity of positive quadratic forms. Proc. Roy. Soc. Edinburgh Sect. A 79 (1977), 267-273.

[Sim78] - A canonical decomposition for quadratic forms with applications to monotone convergence theorems. J. Funct. Anal. 28 (1978), 377-385.

[Stu95] Sturm, K.-T., Analysis on local Dirichlet spaces. II. Upper Gaussian estimates for the fundamental solutions of parabolic equations. Osaka J. Math. 32 (1995), $275-312$.

[Stu98] — The geometric aspect of Dirichlet forms. In New directions in Dirichlet forms, vol. 8 of AMS/IP Stud. Adv. Math., 233-277. Amer. Math. Soc., Providence, RI, 1998.

[Var67a] Varadhan, S. R. S., Diffusion processes in a small time interval. Comm. Pure Appl. Math. 20 (1967), 659-685.

[Var67b] - On the behavior of the fundamental solution of the heat equation with variable coefficients. Comm. Pure Appl. Math. 20 (1967), 431-455. 